\title{
Macamide B Pretreatment Attenuates Neonatal Hypoxic-Ischemic Brain Damage of Mice Induced Apoptosis by Regulates Autophagy Via The PI3K/AKT Signaling Pathway
}

\author{
Xiaoxia Yang \\ Guangdong Pharmaceutical University https://orcid.org/0000-0003-3618-510X \\ Mengxia Wang \\ Guangdong No.2 Provincial People's Hospital \\ Qian Zhou \\ Guangdong Pharmaceutical University \\ Yanxian Bai \\ Guangdong Pharmaceutical University \\ Jing Liu \\ Guangdong Pharmaceutical University \\ Junhua Yang \\ Guangdong Pharmaceutical University \\ Lixia Li \\ Guangdong Pharmaceutical University \\ Guoying Li \\ Guangdong Pharmaceutical University/Guangdong Medical Association \\ Li Luo ( $\sim$ josephluoli@hotmail.com ) \\ Guangdong Pharmaceutical University/Guangdong Medical Association
}

\section{Research Article}

Keywords: Macamide B, Neonatal hypoxic-ischemic brain damage, PI3K/AKT, Autophagy, Apoptosis, Neuroprotecti

Posted Date: October 5th, 2021

DOI: https://doi.org/10.21203/rs.3.rs-952715/v1

License: (c) (1) This work is licensed under a Creative Commons Attribution 4.0 International License. Read Full License 
Version of Record: A version of this preprint was published at Molecular Neurobiology on February 22nd, 2022. See the published version at https://doi.org/10.1007/s12035-022-02751-4. 


\section{Abstract}

Lepidium meyenii (Maca) is an annual or biennial herb from South America that is a member of the genus Lepidium L. in the family Cruciferae. This herb has antioxidant, anti-apoptotic, and enhances autophagy functions and can prevent cell death, and protect neurons from ischemic damage. Macamide $B$, an effective active ingredient of maca, has a neuroprotective role in neonatal hypoxic-ischemic brain damage (HIBD), and the underlying mechanism of its neuroprotective effect is not yet known. The purpose of this study is to explore the impact of macamide B on HIBD-induced autophagy and apoptosis and its potential mechanism for neuroprotection. The modified Rice-Vannucci method was used to induce HIBD on 7-day-old (P7) macamide B and vehicle-pretreated pups. TTC staining was used to evaluate the cerebral infarct volume of pups, brain water content was measured to evaluate the neurological function of pups, neurobehavioral testing was used to assess functional recovery after HIBD, TUNEL and FJC staining was used to detect cell autophagy and apoptosis, and western blot analysis was used to detect the expression levels of the pro-survival signaling pathway phosphatidylinositol-3kinase/protein kinase B (PI3K/AKT) and autophagy and the apoptosis-related proteins. The results show that macamide $B$ pretreatment can significantly decrease brain damage, improve the recovery of neural function after HIBD. At the same time, macamide B pretreatment can induce the activation of PI3K/AKT signaling pathway after HIBD, enhance autophagy, and reduce hypoxic-ischemic (HI)-induced apoptosis. In addition, 3-methyladenine (3-MA), an inhibitor of PI3K/AKT signaling pathway, significantly inhibits the increase in autophagy levels, aggravates Hl-induced apoptosis, and reverses the neuroprotective effect of macamide B on HIBD. Our data indicate that macamide B pretreatment might regulate autophagy through PI3K/AKT signaling pathway, thereby reducing HIBD-induced apoptosis and exerting neuroprotective effects on neonatal HIBD. Macamide B may become a new drug for the prevention and treatment of HIBD.

\section{Introduction}

Hypoxic-ischemic brain damage (HIBD) is a brain lesion produced by hypoxic-ischemic ( $\mathrm{HI}$ ) of brain tissue due to various causes, which can lead to acute death and chronic nerve damage in infants. In developed countries, HIBD occurs in 1-8 newborns out of every 1,000 newborns. In developing countries, up to 26 newborns out of every 1,000 suffer from HIBD disease [1, 2]. Severe HIBD can lead to neurological sequelae such as mental retardation, cerebral palsy and cognitive impairment in infants, and even death. [3]. It is estimated that HIBD causes more than 1 million deaths every year worldwide [4]. To date, mild hypothermia therapy is considered the only neuroprotective treatment that can improve the outcome of neonatal HIBD patients, but only $40 \%$ of patients survive with normal nervous system development [5]. Therefore, the study of HIBD's pathological mechanism and the search for active and effective drugs for neurofunctional repair have become urgent subjects in perinatal medical research.

Lepidium meyenii (Maca) is an annual or biennial herb from South America that is a member of the genus Lepidium $L$. of the family Cruciferae. It is commonly used to treat degenerative diseases, such as neurodegenerative diseases, cardiovascular diseases, diabetes, cancer, and ageing. Maca can remove 
free radicals and protect cells from developing oxidative stress [6-8]. Studies have shown that maca improves the cognitive function of middle-aged mice by up-regulating autophagy-related proteins [9]. Macaamide is the product of maca after drying treatment. It is produced by the formation of amide bonds between different amino-containing compounds and various fatty acids. Macamides are part of the unique phytochemical composition of maca, which is rich in nutrients and has remarkable medicinal effect. It has the functions such as anti-oxidation, anti-inflammatory, anti-osteoporosis, memory improvement, nerve cell protection, and nervous system regulation [9-12]. However, Macamide B (structure shown in Fig. 1a) is an effective monomer of macamide, and its potential mechanism in providing neuroprotection and exerting neuroprotective effects is not yet known.

The phenotypes of autophagy, apoptosis, oxidative stress, inflammation and necrosis are closely related to the pathological development of HIBD $[13,14]$. Among these factors, accumulated data indicate that the mechanism of autophagy and apoptosis plays a fundamental role in ischemic brain damage in newborn rodents [13-15]. Currently, many studies have focused on autophagy and apoptosis as a therapeutic target to prevent neonatal $\operatorname{HIBD}[13,16,17]$. Autophagy is a process that widely exists in eukaryotic cells, and uses the lysosomal pathway to degrade self-damaged organelles, proteins and macromolecular substances. It maintains cell survival by degrading and restoring over-expressed proteins and organs in cells. It has been considered to be related to brain ageing and various neurodegenerative diseases are related [18]. Stroke studies have shown that autophagy damage can aggravate the nerve damage induced by cerebral ischemia [19]. In the study of ischemia-reperfusion (I/R) damage, melatonin played a neuroprotective role on I/R damage by activating autophagy [20]. In addition, in focal cerebral ischemia and glucose and oxygen deprivation (OGD) models, autophagy has a neuroprotective effect on glial cells [21]. The death and survival of cells usually require the co-regulation of apoptosis and autophagy. Reducing neuronal apoptosis is a crucial process in determining neural functional recovery after HIBD. In the I/R model, the intervention of electroacupuncture significantly inhibits neuronal apoptosis induced by cerebral ischemia, thereby reducing the brain damage after I/R [22]. Acute spinal cord damage studies have found that inhibiting autophagy significantly aggravates cell apoptosis in the spinal cord damage area and hinders the recovery of nerve function after spinal cord damage [17]. As an effective active ingredient of Maca, Macamide B can prevent neonatal HIBD by regulating autophagy and reducing nerve cell apoptosis. It is worthy of further investigation.

The phosphatidylinositol-3-kinase/protein kinase B (PI3K/AKT) pathway is one of the most crucial signaling cascade that regulate autophagy, apoptosis, proliferation, and growth of mammalian cells (Fig. 1b). It plays a vital role in mediating the pathological process of nervous system injury. In the treatment of neurodegenerative diseases, PI3K/Akt pathway is considered to be a target to promote cell survival [23]. Therefore, the neuroprotective effect of PI3K/Akt pathway in cerebral ischemia has been extensively studied. Studies have shown that Geniposide can protect perinatal HI-induced brain damage by activating the PI3K/Akt signaling pathway [24]. Ginkgetin can inhibit cell apoptosis induced by I/R by activating PI3K/Akt pathway [25]. In the rat middle cerebral artery occlusion (MCAO) model, Sodium butyrate inhibits neuronal apoptosis through GPR41/Gbc/PI3K/Akt signaling pathway, thereby reducing brain damage after MCAO and improving functional outcome [26]. Increased nerve cell apoptosis is a 
common consequence of HIBD, and reducing cell apoptosis is essential to improve the neurological dysfunction caused by HIBD. As we all know, the PI3K/Akt pathway is also a meaningful way to regulate autophagy. PI3K/Akt pathway inhibitors can significantly inhibit the expression of autophagy-related proteins induced by HBCDs, suggesting that activation of PI3K/Akt pathway may promote the increase of HBCDs-induced autophagy levels [27]. It has been reported that melatonin reduces neuronal damage caused by I/R by activating the expression of autophagy-related levels [28]. In addition, the induction of mitochondrial autophagy through endoplasmic reticulum stress can prevent transient ischemic brain damage [29]. This indicates that activating the PI3K/Akt pathway to enhance autophagy may be a promising therapeutic strategy for HIBD to induce apoptosis.

$\mathrm{PI3K} /$ Akt signaling pathway plays a vital role in the process of cell autophagy and apoptosis [30]. A therapeutic strategy dedicated to activating the PI3K/Akt signaling pathway to enhance autophagy, thereby reducing ischemic penumbra (IP) cell apoptosis seems very promising. However, the ability of macamide $B$ to ameliorate HIBD in neonatal mice, and whether macamide $B$ pretreatment can regulate autophagy through the PI3K/AKT signaling pathway after HIBD, thereby reducing HIBD-induced apoptosis is unclear. Therefore, this study explored the potential of macamide $B$ to regulate autophagy through PI3K/AKT signaling pathway to reduce HIBD in neonatal mice and inhibit cell apoptosis.

\section{Materials And Methods}

\section{Animals}

The study selected P7 C57BL/6 pups (male or female), weighing 3 to $5 \mathrm{~g}$, provided by the Guangdong Medical Laboratory Animal Center (Guangzhou, Guandong). Each squirrel cage allows one female mouse to take care of its pups. Mice are reared in an environment with a temperature of 20 to $24^{\circ} \mathrm{C}$ and a humidity of $40 \%$ to $70 \%$. All animal-related experiments done in this study have been approved by the Experimental Laboratory Animal Committee of Guangdong Pharmaceutical University and comply with the Chinese Council on Animal Care guidelines.

\section{HIBD model and macamide $B$ administration}

In this experiment, we used a total of 160 P7 C57BL/6 pups. The improved Rice-Vannucci method was used to construct the HIBD model [31, 32]. Briefly, C57BL/6 pups at 7-9 days of age (P7-9) were given continuous inhalation anaesthesia (isoflurane) through a face mask, fixed on a sterile surgical drape in the supine position, and disinfected at the predetermined surgical incision location on the neck skin. A 1 $\mathrm{cm}$ incision was cut along the middle of the neck, the subcutaneous tissue was bluntly separated, and the unilateral common carotid artery (CCA) was separated, a coagulator was used to cut off the CCA, the skin was sutured and disinfected, and the pups were returned to the dams for feeding and recovery. After recovering for $1 \mathrm{~h}$, put the pups in a $37^{\circ} \mathrm{C}$ water bath controlled hypoxia box, and a mixed gas of oxygen 
(8\%) and nitrogen (92\%) was supplied. The pups were exposed to hypoxia for $4 \mathrm{~h}$ then returned to the dams to feed, and the model was completed.

Macamide B (Shanghai yuanye Bio-Technology Co., Ltd, China, HPLC $\geq 98 \%$ ) was dissolved in PBS solution $(1.25 \mathrm{mg} / \mathrm{ml})$. Twenty minutes before the ischemia surgery, the macamide $B$ group mice were intraperitoneally injected with macamide $B(60 \mathrm{mg} / \mathrm{kg})$. For pups in the vehicle group, give the same volume of PBS treatment. The protocol is described in Fig. 1c.

\section{Infarct volume measurement}

After HIBD for $24 \mathrm{~h}$, intraperitoneal administration of $10 \%$ chloral hydrate to the pups for anaesthesia $(0.1$ $\mathrm{ml}$ ), and the brains were immediately extracted. We cut the pup's brain into four brain slices in a coronal plane (the intervals between adjacent brain slices is $2 \mathrm{~mm}$ ). The sections were stained for $20 \mathrm{~min}$ in a $2 \%$ solution of 2,3,5-triphenyltetrazolium chloride (TTC, Sigma-Aldrich, Germany), turning the brain slices from time to time to make even contact with the staining solution. Then the images of the brain slice of the pup were captured by a digital camera. The nonischemic necrotic area was red, while the ischemic necrotic tissue was white. Use ImageJ software (version 1.8.0, USA) to analyze the cerebral infarct volume of pups. The percentage of infarct volume $=[($ contralateral hemisphere - un-infarcted area of ipsilateral hemisphere)/contralateral hemisphere × 2] × 100\% [1].

\section{Measurement of brain water content}

After HIBD for $24 \mathrm{~h}$, intraperitoneal administration of $10 \%$ chloral hydrate to the pups for anaesthesia $(0.1$ $\mathrm{ml}$ ), and the brains were immediately extracted. The pup's brain was divided into two parts and weighed for wet weight and the cerebral hemispheres were dried in an oven at $106^{\circ} \mathrm{C}$ for $24 \mathrm{~h}$ and weighed for dry weight. Percentage of brain water content $=$ (wet weight - dry weight $) /$ wet weight $\times 100 \%$. The percentage of brain water content $=$ (wet weight - dry weight) $/$ wet weight $\times 100 \%$.

\section{Neurobehavioral assessments}

Neurological damage caused by HIBD can lead to sensorimotor impairments. Body weight and sensorimotor performance (righting reflex, negative geotaxis, and grip test) were tested 1, 3, and 7 days after the $\mathrm{HI}$ procedure in a blinded manner [33].

\section{Righting reflex}

The righting reflex is used to evaluate the recovery of the brain of pups. The pups were placed on a flat surface with one hand gently holding the head and the other hand gently holding the hind limbs; the pup was rolled onto its back and released. The time required for the mouse to return to an upright position (all 
limbs on the ground) was recorded. The maximum testing time was $1 \mathrm{~min}$, and times over 1 min were recorded as $1 \mathrm{~min}$.

\section{Negative geotaxis}

The geotaxis test was used to diagnose vestibular or proprioceptive functions. Place the pup on a flat surface inclined at 45 degrees, with the head of the pup facing the bottom of the plane, and record the time it takes for the head and body of the pup to make a $180^{\circ}$ turn. The maximum testing time was 1 min, and times over $1 \mathrm{~min}$ were recorded as $1 \mathrm{~min}$.

\section{Grip test}

The pup grasped a metal wire with a diameter of $1.5 \mathrm{~mm}$ with both front feet. The metal wire was stretched horizontally in a test box with a width of $50 \mathrm{~cm}$. The distance between the metal wire and the bottom of the box was $15 \mathrm{~cm}$. The bottom was covered with cork chips. Record the time it takes for the pup to grasp the wire to release it. The minimum testing time was $20 \mathrm{~s}$, and times below $20 \mathrm{~s}$ were recorded as $20 \mathrm{~s}$.

\section{Western blot analysis}

Protein samples $(15 \mu \mathrm{l} /$ well) were separated on SDS-PAGE gel ( $80 \mathrm{~V}, 110 \mathrm{~min})$ and transferred to 0.22 $\mu \mathrm{m}$ polyvinylidene difluoride (PVDF) membrane (290 mA, $100 \mathrm{~min}$ ). Five percent nonfat dry milk in TBST buffer was used to block nonspecific sites for $1.5 \mathrm{~h}$. Incubate the primary antibody in a refrigerator at $4^{\circ} \mathrm{C}$ for $16 \mathrm{~h}$. Use that following primary anti-antibody: PI3K (1:1500, Abcam, UK), p-PI3K (1:1500, Abcam, UK), AKT (1:1500, Proteintech, USA), p-Akt (1:1500, Cell Signaling Technology, USA), Beclin1 (1:750, Abcam, UK), LC3B (1;500, Proteintech, USA), p62 (1:1000, Abcam, UK), p53 (1:500, Multisciences, China), Bax (1:750, Proteintech, USA), Bcl-2 (1:750, Proteintech, USA), caspase-3 (1:600, Proteintech, USA). and cleaved caspase-3 (1:650, Proteintech, USA). $\beta$-actin (1:800; Proteintech, USA) was employed as an internal reference protein. After $16 \mathrm{~h}$, the corresponding secondary antibodies (1:6000, EarthOx, USA) were incubated for $90 \mathrm{~min}$. Bands were measured by an automatic chemiluminescence image analysis system (Tanon 5200, Shanghai, China). ImageJ software was used to carry out Western blot analysis, and SPSS 21.0 was applied to process the data.

\section{Terminal deoxynucleotidyl transferase dUTP nick end labeling (TUNEL) staining.}

Because cells are prone to programmed cell death during HI [34]. Therefore, we assessed the apoptotic cells in the brain tissue of the pups by TUNEL Apoptosis Detection Kit (fluorescence) (Wanleibio, China). 
On day three after $\mathrm{HI}$ damage, the pups were perfused into the heart, and fresh brain tissue was quickly collected in $4 \%$ paraformaldehyde (PFA) for fixation. After fixation for $24 \mathrm{~h}$, the brain tissue of the pup was rinsed under running water for $16 \mathrm{~h}$. After washing with running water, perform gradient dehydration, paraffin embedding, tissue sectioning and other operations on the brain tissue of pups for subsequent TUNEL detection.

The slices were immersed in xylene $\nabla$ and $\nabla$ for 15 min each, immersed in $100 \% \otimes, 100 \% \otimes$, and $95 \%, 90 \%$, $80 \%, 70 \%$, and $50 \%$ ethanol solutions for 5 min per solution, and washed with $\mathrm{ddH}_{2} \mathrm{O}$ for $3 \times 5 \mathrm{~min}$. Put that slices into citrate buffer solution $(0.01 \mathrm{~mol} / \mathrm{L})$, and place them in a microwave oven at hightemperature repair for $20 \mathrm{~min}$. Add $25 \mu \mathrm{l}$ of $3 \% \mathrm{H}_{2} \mathrm{O}_{2}$ buffer to each brain tissue and let stand for $12 \mathrm{~min}$ in the dark, followed by PBS washing for $3 \times 5 \mathrm{~min}$. Then, add $25 \mu$ T TUNEL reaction buffer to each brain tissue and incubated in a $37^{\circ} \mathrm{C}$ incubator in the dark for $90 \mathrm{~min}$. After washing with PBS for $3 \times 5 \mathrm{~min}$, counter-stain brain tissue cell nuclei with DAPI for $5 \mathrm{~min}$, then repeat the PBST wash step. After absorbing excess water with filter paper, add $25 \mu \mathrm{l}$ of anti-fluorescence quencher to each brain tissue for mounting, immediately observe with a fluorescence microscope (Olympus BX51, Japan), or store it at $-20^{\circ} \mathrm{C}$ for observation within a week.

\section{Fluoro-Jade C Staining}

After HIBD for $24 \mathrm{~h}$, the pups were immediately sacrificed, and brain paraffin sections were made. Fluoro-Jade C (FJC) staining (Biosensis, USA) was used to detect the degeneration of neurons in the pup's brain tissue.

The slices were immersed in xylene $₫$ and $\nabla$ for 15 min each, immersed in $100 \% \rrbracket$ and $100 \%$ ethanol solutions for 5 min per solution, immersed in $70 \%$ ethanol solutions for 5 min per solution, and washed with $\mathrm{ddH}_{2} \mathrm{O}$ for $2 \times 5 \mathrm{~min}$. Transfer slides to a new Coplin jar containing $\mathrm{ddH}_{2} \mathrm{O}$ for $2 \mathrm{~min}$. Mix 9 parts of $\mathrm{ddH}_{2} \mathrm{O}$ and 1 part of potassium permanganate solution and add to the brain tissue slices and incubate at room temperature for 10 min. Rinse slides for 2 min in $d_{d d H_{2}} \mathrm{O}$. Mix 9 parts of $d_{d d H_{2}} \mathrm{O}, 1$ part of FJC solution and 1 part of DAPI solution and add to the brain tissue slices and incubate for $12 \mathrm{~min}$. Wash in $\mathrm{ddH}_{2} \mathrm{O} 3 \times 1 \mathrm{~min}$. Put the slices in a $56^{\circ} \mathrm{C}$ oven and bake for $5 \mathrm{~min}$. The dry slides are then cleared by brief (5 min) immersion in xylene. Mount the slides with an anti-fluorescence quencher. Immediately observe with a fluorescence microscope, or store it at $-20^{\circ} \mathrm{C}$ for observation within a week.

\section{Tissue immunofluorescence staining}

After HIBD for $24 \mathrm{~h}$, the pups were immediately sacrificed, and brain paraffin sections were made. Tissue immunofluorescence staining was used to detect the expression levels of p53, Bax, Bcl-2, caspase-3, and cleaved caspase- 3 in the brain tissue of mice. 
Repeat tissue dewaxing and high-temperature repair steps, and incubate with Quick Block ${ }^{\mathrm{TM}}$ immunostaining blocking solution for $20 \mathrm{~min}$. Incubate each brain tissue section with the primary antibody at $4^{\circ} \mathrm{C}$ for $16 \mathrm{~h}$, and add $0.5 \%$ Triton X-100 to the corresponding primary antibody for cell rupture. The following primary antibodies were used:Beclin1 (1:200, Abcam, UK), LC3B $(1 ; 250$, Proteintech, USA), p62 (1:260, Abcam, UK), p53 (1:260, Multisciences, China), Bax (1:180, Proteintech, USA), Bcl-2 (1:260, Proteintech, USA), caspase-3 (1:280, Proteintech, USA), and cleaved caspase-3 (1:280, Proteintech, USA). After $16 \mathrm{~h}$, the membranes were removed from the refrigerator, allowed to return to room temperature for $30 \mathrm{~min}$, and washed again with PBST for $3 \times 10$ min. Dylight 488 labelled goat antirabbit fluorescent secondary antibody (1:360, Sigma-Aldrich, USA) or Dylight 594 labelled goat anti-rabbit fluorescent secondary antibody (1:360, Sigma-Aldrich, USA), incubate for $2 \mathrm{~h}$ at room temperature in the dark. After washing with PBS for $3 \times 5$ min, counter-stain brain tissue cell nuclei with DAPI for 5 min, then repeat the PBST wash step. Mount the slides with an anti-fluorescence quencher. Immediately observe with a fluorescence microscope, or store it at $-20^{\circ} \mathrm{C}$ for observation within a week.

\section{Statistical analysis}

All of the experiments were repeated at least three times. The data are presented as the mean \pm SEM. Statistical analyses were carried out by SPSS.21.0 and GraphPad Prism (version 8.0, USA). Differences between individual groups were first compared using analysis of variance (one-way ANOVA), and then post hoc testing was analyzed with Tukey or Student-Newman-Keuls multiple comparisons. Difference between the two groups were compared using Student's t-test. A $p<0.05$ indicated that the difference between the two groups was statistically significant.

\section{Results}

\section{Macamide B pretreatment attenuated HIBD in newborn mice}

In order to determine the optimal dose of macamide B pretreatment to treat neonatal HIBD, three doses were used for dose exploration: low dose (30 mg/kg), medium dose $(60 \mathrm{mg} / \mathrm{kg})$, and high dose (90 $\mathrm{mg} / \mathrm{kg}$ ). The results of TTC staining (Fig. 2a, b) showed that, compared with the vehicle group, the medium dose $(60 \mathrm{mg} / \mathrm{kg})$ of macamide $B$ significantly reduced the percentage of infarct volume $(p<0.05)$. Compared with the vehicle group, there was no significant difference in infarct volume between the lowdose $(30 \mathrm{mg} / \mathrm{kg})$ and high-dose $(90 \mathrm{mg} / \mathrm{kg})$ groups ( $>0.05)$. It shows that the pretreatment of macamide $B$ at a medium dose $(60 \mathrm{mg} / \mathrm{kg})$ can significantly reduce the cerebral infarction volume of HIBD pups.

Brain water content was used to assess brain edema damage in pups, as shown in the figure (Fig. 2c). The water content of the ipsilateral cerebral hemisphere in the vehicle group was significantly higher than that in the sham group $(p<0.0001)$. Macamide B pretreatment significantly reduced the water content of the ipsilateral cerebral hemisphere in the vehicle group $(p<0.0001)$. The above results indicate that 
macamide B pretreatment significantly reduces HI-induced brain damage and has neuroprotective effects on HIBD in newborn mice.

\section{Macamide B pretreatment improved neurobehavioral performance after neonatal HIBD}

To explore whether macamide B can promote general health and recovery of neurological function, we conducted the following neurobehavioral tests at 1,3, and 7 days after surgery: righting reflex, negative geotaxis, and grip tests in the sham, vehicle, and macamide $B$ groups. Body weight is used to evaluate general health. At 1,3 , and 7 days after HIBD, macamide $B$ pretreatment can significantly reverse the weight loss of pups after HIBD compared with the vehicle group (Fig. 3a). In the righting reflex (Fig. 3b), negative geotaxis (Fig. 3c), and grip tests (Fig. 3d), mice in the macamide B group exhibited superior performance at 1,3 , and 7 days after surgery than those in the vehicle group. These outcomes indicated that macamide B could improve neurobehavioral performance following HIBD.

\section{Macamide B pretreatment activates PI3K-AKT signaling pathway}

Whether the PI3K-AKT signaling pathway is involved in the neuroprotective mechanism of macamide B after $\mathrm{HI}$ damage is not yet known. Western blot was used to detect the expression levels of proteins related to the PI3K-AKT signaling pathway. Western blot experiment results showed that compared with sham, the expression levels of p-PI3K and p-AKT in the vehicle group were significantly decreased ( $p$ $<0.0001$, Fig. $4 b, c)$. Compared with the vehicle group, macamide B pretreatment significantly increased the expression levels of p-PI3K and p-AKT protein $(p<0.0001$, Fig. $4 b, c)$. Western blot showed that macamide B pretreatment significantly activates the PI3K-AKT signaling pathway. Macamide $B$ has a neuroprotective effect on HIBD in neonatal mice, which may be mediated by the PI3K-AKT signaling pathway.

\section{Macamide B pretreatment significantly enhances the autophagy level of HIBD neonatal mice}

To evaluate the effect of macamide B pretreatment on autophagy. Tissue immunofluorescence staining and western blot were used to detect the expression levels of autophagy-related proteins Beclin1 (Fig. 5a), LC3B (Fig. 5b), and p62 (Fig. 5c). Tissue immunofluorescence staining results showed that after HIBD, the number of Beclin 1 and LC3B positive cells was significantly reduced, and the number of p62 positive cells was significantly increased. Macamide B pretreatment reversed this result. The results of western blot experiments showed (Fig. 6) that, compared with sham, the expression levels of Beclin1 and LC3B in the vehicle group were significantly decreased $(p<0.0001)$, and the expression levels of p62 were significantly 
increased $(p<0.001)$. After pretreatment with macamide $B$, macamide $B$ significantly up-regulated the expression levels of Beclin 1 and LC3B $(p<0.0001 ; p<0.05)$, and down-regulated the expression levels of p62 ( $p<0.01)$. Tissue immunofluorescence staining and western blot results indicate that macamide $B$ pretreatment promotes autophagy, and macamide B may exert neuroprotective effects on HIBD in neonatal mice by enhancing autophagy.

\section{Macamide B pretreatment significantly reduces HIBD- induced apoptosis}

In order to detect the effect of macamide B pretreatment on cell apoptosis of HIBD neonatal mice. TUNEL and FJC staining were used to evaluate neuronal apoptosis and degeneration. Tissue immunofluorescence staining and western blot were used to detect the expression levels of apoptosisrelated proteins $\mathrm{p} 53, \mathrm{Bax}, \mathrm{Bcl}-2$, caspase-3, and cleaved caspase-3.

\section{3-MA inhibits the PI3K-AKT signaling pathway and reverses the neuroprotective effect of macamide B on HIBD in neonatal mice}

3-MA is a selective inhibitor of PI3K and has an inhibitory effect on class III PI3K [35]. To determine whether macamide $B$ exerts a neuroprotective effect on HIBD in neonatal mice through the PI3K-AKT signaling pathway. After treatment with 3-MA, an inhibitor of the PI3K-AKT signaling pathway in HIBD newborn mice, 3-MA significantly inhibited p-PI3K, p-AKT protein expression (Fig. 9c, d; $p<0.0001$ ), and blocked the activation of PI3K-AKT signaling pathway induced by macamide $B$ pretreatment. At the same time, the results of the TTC experiment showed (Fig. 9a, b) that compared with the macamide B + vehicle 2 group, the 3-MA intervention significantly increased the cerebral infarction area of the pups $\mathbb{p}<0.0001 \rrbracket$; The brain water content measurement results showed (Fig. 2c) that compared with the macamide B + vehicle 2 group, 3-MA intervention significantly aggravated the brain edema damage of pups $(p<0.001)$; In the behavioural experiment results, compared with the macamide B + vehicle 2 group, 3-MA intervention significantly aggravated the weight loss of HIBD neonatal mice at 1,3 , and 7 days after HIBD (Fig. 9c), Macamide exerts a neuroprotective effect on HIBD in newborn mice through the PI3K-AKT signaling pathway.

\section{3-MA treatment inhibits autophagy and reverses the protective effects of Macamide B pretreatment on apoptosis}

3-MA is an inhibitor of the PI3K-AKT signaling pathway, and is also a well-known autophagy inhibitor, which works by inhibiting the formation of autophagosomes [35]. In our study, immunofluorescence (Fig. 
10) showed a significant increase in Beclin1 and LC3B positive cells and a significant decrease in $p 62$ positive cells after 3-MA intervention compared to the macamide $B+$ vehicle group. The results of western blot (Fig. 11) were consistent with the trend of immunofluorescence results. 3-MA intervention significantly inhibited the expression levels of Beclin1 and LC3B proteins $(p<0.0001)$, and up-regulated the expression level of p62 protein $(p<0.001)$. In apoptosis-related studies, fluorescence experiments (Fig. 12) showed that 3-MA intervention resulted in a significant increase in the number of TUNEL-positive cells, FJC-positive neurons, and p53, Bax, caspase-3, and cleaved caspase-3 cells, and a significant decrease in the expression of $\mathrm{Bcl}-2$ positive cells, compared with the macamide $\mathrm{B}+$ vehicle group. The results of western blot experiments showed (Fig. 13) that compared with the macamide B + vehicle group, 3-MA intervention significantly increased the expression levels of $p 53$, caspase- 3 and cleaved caspase-3 $(p<0.0001 ; p<0.001)$, and reduced $\mathrm{Bcl} 2$ / Bax ratio $(p<0.0001)$. The above results indicate that macamide $B$ pretreatment may enhance autophagy by activating the PI3K-AKT signaling pathway, thereby reducing HIBD-induced apoptosis.

\section{Discussion}

Neonatal HIBD is one of the common causes of neonatal death and disability. This condition usually causes sequelae such as cerebral palsy, visual impairment, and mental retardation [36, 37]. Mild hypothermia therapy is considered to be an effective method to reduce the mortality of HIBD. However, the disability and mortality rate of HIBD is still high [38]. At the same time, due to limitations in equipment, human and financial resources, and other objective reasons, mild hypothermia therapy is not yet entirely popularized in developing countries. Therefore, finding new safe and effective therapeutic drugs is the primary task of HIBD research. As a precious herb, maca has been proven to have neuroprotective effects in previous studies $[9,10]$. However, the potential therapeutic impact of maca's effective monomer, macamide $B$, in providing neuroprotection in HIBD has remained unclear, and the key pathways and potential mechanisms of its neuroprotective effects are not yet known.

In this study, we confirmed that Macamide B has a neuroprotective effect on HIBD in newborn mice. Specifically, we showed that (1) macamide B pretreatment significantly reduces HI-induced brain damage, (2) pretreatment with macamide B can significantly improve neurobehavioral results after HIBD, (3) macamide B pretreatment significantly activates the PI3K/Akt signaling pathway, enhances autophagy, and inhibits cell apoptosis, and (4) the neuroprotective effect of macamide B may be through the $\mathrm{PI} 3 \mathrm{~K} / \mathrm{Akt}$ signaling pathway to regulate autophagy, thereby reducing HIBD-induced apoptosis. In this study, we clarified that macamide B pretreatment protects neonatal mouse brains from HIBD and improves general conditions and neurobehavior.

The treatment and prevention of neonatal HIBD have always attracted much attention, but due to the complexity of the nervous system, treating this disease poses severe challenges. Medicinal plants can be advantageous in the drug discovery process because they have undergone indirect clinical trials during their long-term use, and their side effects and toxicity are usually known. Given the complexity of neurodegenerative diseases, natural drugs may be good candidates for targeting phenotypes such as 
apoptosis, autophagy, oxidative stress, and inflammation and promote the recovery of cell death and neuronal damage in focal ischemic stroke and many neurodegenerative diseases $[39,40]$. Maca is a rare medicinal and edible plant that originated in the central part of the Peruvian Andes. It has rich nutritional value and can relieve fatigue, improve sleep, and improve sexual function and exhibits antioxidant activity. Maca is called "Peruvian Ginseng" [41-43]. Studies have shown that maca has extensive neuroprotective effects both in vivo and in vitro. The cerebral infarct volume of mice with neurons damaged by $\mathrm{H}_{2} \mathrm{O}_{2}$ was treated with maca is significantly reduced in mice treated with maca, and antiapoptosis, antioxidation, prevention of cell death, and protection of neurons from ischemia damage are its primary mechanisms of action [9]. In vitro studies showed that the viability of crayfish neurons was significantly enhanced by treatment with maca extract, which also showed the neuroprotective effects of maca [9]. Maca can be used as a neuroprotective agent alone or synergistically with other protective agents to prevent neurodegeneration and cell death in stroke and other neurodegenerative diseases [44]. Autophagy disorders are thought to be related to brain ageing and a variety of neurodegenerative diseases. Maca can promote the up-regulation of autophagy-related proteins by activating the autophagy signal in the mouse cortex and improve the cognitive function of middle-aged mice [23]. In 2000, Zheng $B L$ and others first discovered the unique active substance macamide in maca [44]. Macamide, a type of benzylated or 3-methoxybenzylated alkanamide alkaloid, is a unique secondary metabolite in maca [44]. Purified macamide and synthetic analogues of macamide can exert neuroprotective effects by acting on the endocannabinoid system [45]. Studies have used a zebrafish model of dopaminergic neuron loss to evaluate the neuroprotective effect of macamide. It was found that macamide extract exerts a significant neuroprotective effect on zebrafish neurons [46]. Whether macamide B, a unique monomer of macamide, has a neuroprotective effect in neonatal HIBD was investigated in this study, which also included an indepth study of the potential mechanism of its neuroprotective effect.

In this study, one of the issues we need to consider is the effectiveness of the route of administration of macamide $B$ in the central nervous system (CNS). The prerequisite for the direct effect of drugs on the CNS is that the drug must first pass through the blood-brain barrier (BBB) from the blood and enter the extracellular fluid of the CNS to be effective. [47]. Therefore, it is necessary to confirm whether macamide $B$ can pass through the BBB. Studies have shown that the lipophilicity of certain components of maca can promote their passage through the BBB and affect CNS function. Related reports have shown that some ingredients in maca are active in the CNS [48-50]. For some compounds, the neonatal BBB is more permeable than the adult BBB [51]. At the same time, it has also been reported that when pups are injured by $\mathrm{HI}$, the normal function of the BBB will be destroyed, which will increase the permeability of the BBB. This situation also increases the possibility of macamide $B$ successfully reaching the brain through the BBB [51]. Besides, pups have a large peritoneal surface area and strong exchange capacity, and intraperitoneal administration of macamide $B$ enabled macamide $B$ to reach the brain through different signal transduction pathways and thus exert its neuroprotective effect. We used the Rice-Vannucci method to establish a neonatal mouse HIBD model by ligating one side of the common carotid artery of the pups to cause hemi-brain tissue ischemia and then placing the pups in a closed hypoxic tank for $4 \mathrm{~h}$. This model is currently recognized as an ideal model for the study of neonatal HIBD $[52,33]$. The results 
of TTC staining showed that there was a clear cerebral infarction area on the ligation side of the brain, which verified that the brain tissue damage caused by surgery and hypoxia was, as proposed by Towfghi $\mathrm{J}$ et al., mainly confined to the carotid artery ligation side [53], these results also indicate that the HIBD model was successfully constructed. Compared with the vehicle group, the cerebral infarct volume of pups pretreated with macamide $B$ was significantly reduced, indicating that macamide $B$ has a significant neuroprotective effect on HIBD in newborn mice. In this experiment, P7 pups were selected as the model mice, mainly because the brains of P7 mouse pups are similar in histological structure to the 32-34 week fetus or newborn [54].

Increasing evidence has shown that apoptosis is a crucial pathological trigger involved in neurological deficits after HIBD [55]. Compared to adult brains, apoptosis is more common in immature newborn brains [56]. Inhibition of neuronal apoptosis is strongly recommended as a therapeutic target for neuronal rescue in the neonatal HIBD paradigm [57]. Studies have found that the tumor suppressor p53 may trigger the apoptosis pathway after DNA damage, and activate caspase 3 to induce cell death by upregulating the expression of the pro-apoptotic protein Bax [58]. In our study, macamide B pretreatment significantly reduces the expression level of $\mathrm{p} 53$, and has a regulatory effect on the apoptotic pathway. Apoptosis is a kind of programmed cell death that occurs through regulating genes and their products in cells. Reducing apoptosis is an essential component in the recovery of neurological function in mammals with brain damage. Caspase- 3 is a critical protein in the apoptosis signaling pathway, which can induce cell apoptosis in animal models of ischemic stroke [59]. In I/R research, caspase-3 is a key protein involved in the inflammation and apoptosis of $\mathrm{I} / \mathrm{R}$ damage. Both ischemia and reperfusion lead to an increase in caspase-3 activity [60]. Inhibition of caspase-3 activation can effectively prevent neonatal HIBD [61]. Bcl-2 and Bax are the crucial proteins of the Bcl-2 gene family, $\mathrm{Bcl}-2$ cell apoptosis inhibitor gene, Bax can antagonize the protective effect of $\mathrm{Bcl}-2$ and induce cell apoptosis [62]. Studies have shown that the ratio of $\mathrm{Bcl}-2 / \mathrm{Bax}$ activity levels is a crucial determinant of cell susceptibility to apoptosis, not the level of individual proteins [63]. In the I/R damage of rats, ginkgetin can restore mitochondrial membrane potential by up-regulating the activity level of Bcl-2/Bax in rats with brain damage, thereby inhibiting apoptosis induced by the caspase-3 pathway and playing a neuroprotective role in rat I/R damage [25]. In MCAO rats and the SH-SY5Y cell model induced by oxygen glucose deprivation/reoxygenation (OGD/R), Chrysin reduces the expression levels of Bax and cleaved caspase-3 by increasing the expression level of $\mathrm{Bcl}-2$, reducing the I/R damage and apoptosis of $\mathrm{SH}-\mathrm{SY} 5 \mathrm{Y}$ cells induced by OGD/R. [64]. In our study, macamide B pretreatment can reverse the increase in caspase-3, cleaved caspase-3, and Bax expression levels induced by $\mathrm{HI}$ stimulation, and up-regulate the expression levels of $\mathrm{Bcl}-2$ and the number of TUNEL and FJC positive neurons. Therefore, macamide B may effectively prevent neonatal HIBD through the anti-apoptotic pathway.

Cell death and survival in neurodegenerative diseases are usually regulated by autophagy and apoptosis. Autophagy maintains cell survival by degrading and restoring dysfunctional organelles and misfolded proteins [63]. LC3B is currently the most widely used autophagy marker protein, reflecting the number of autophagosomes. When autophagy occurs, LC3B $\otimes$ is converted to LC3B $\otimes$, accompanied by the formation of autophagosomes. Beclin1 is the first autophagy-promoting protein found in mammals and regulates 
autophagosome-lysosome fusion. P62 (SQSTM1) is the primary substrate for degradation during autophagy and plays a vital role in the aggregation and removal of ubiquitinated proteins. The accumulation of P62 indicates that the initiation of autophagy is decreased or the fusion of autophagosomes and lysosomes is disordered, which is negatively correlated with the level of autophagy activity $[65,66]$. In the I/R model, the remote limbic postconditioning (RIPoC) mitigates I/R damage by activating autophagy [67]. Similarly, Astragaloside IV can play a neuroprotective effect on brain damage caused by ischemic stroke by promoting autophagy [68]. When HIBD occurs, autophagy and apoptosis are often inseparable. The signal network between autophagy and apoptosis is staggered, coherent, and complex, and they affect each other. Studies have shown that mild hypothermia reduces microglia activation after traumatic brain damage by inhibiting autophagy and promoting apoptosis, indicating that apoptosis of autophagy is interrelated [69]. It has been reported that activated autophagy can alleviate I/R damage by inhibiting the apoptosis cascade of ischemic stroke [70]. All these studies show that enhancing autophagy and inhibiting apoptosis has a neuroprotective effect on brain damage. However, it has also been reported that autophagy is harmful to I/R damage, and inhibition of autophagy activation may reduce I/R damage [71]. This controversy may be caused by different ischemia time, animal model, animal strain, administration time, and injection time of inhibitor agonist. Xiaowei Sun et al. found that Eugenol played a neuroprotective role in I/R damage by promoting the increase of Beclin1 level and LC3II/I ratio induced by MCAO or OGD/R, and the decrease of p62 level [72]. Consistent with the research of Xiaowei Sun et al., in this study, we proved that enhancing autophagy is beneficial to HIBD. Macamide B pretreatment significantly promoted the increased of Beclin1 and LC3B expression level and the decrease of p62 level induced by HIBD, reduced brain damage induced by $\mathrm{HI}$, and improved neurological deficit. However, a selective inhibitor of PI3K, also known as the autophagy inhibitor 3-MA, reversed this finding and exacerbated apoptosis. Therefore, macamide $B$ may reduce the apoptosis induced by HIBD by enhancing autophagy, and play a neuroprotective role on HIBD in newborn mice.

$\mathrm{PISK} / \mathrm{AKT}$ is a vital pro-survival signaling pathway, involved in many critical cellular processes, such as apoptosis, autophagy, and proliferation, and is considered to be an important regulator of autophagy and apoptosis. Studies have shown that this signaling pathway can protect neurons from damage from different brain diseases [73, 74]. p-Akt can be observed in both adults and neonates soon after cerebral ischemia, and IP is particularly significant [75]. Studies have shown that activation of PI3K/AKT and its downstream pathways can inhibit neuronal apoptosis [76]. When a brain damage occurs, pAkt can inhibit cell apoptosis, and the increase of pAkt protein has a neuroprotective effect on HIBD $[77,78]$. Xiaohui Tan et al. found that luteolin can reduce neurotoxicity by inhibiting PI3K/Akt pathway-mediated p53 accumulation and p53-triggered apoptotic pathway, and exert neuroprotective effects on rat brain damage [79]. Consistent with the study by Xiaohui Tan et al., in this study, pretreatment with macamide B inhibited the PI3K/Akt pathway through 3-MA, resulting in a large accumulation of p53, thereby aggravating the apoptosis triggered by $\mathrm{p} 53$, leading to caspase -3 , cleaved caspase -3 and Bax expression levels increased, and Bcl-2 expression levels decreased. On the contrary, the PI3K/Akt pathway activated by macamide B pretreatment can antagonize the apoptosis induced by HIBD and play a neuroprotective effect. Recent studies have shown that autophagy has a neuroprotective effect, and 
autophagy can alleviate the traumatic brain injection (TBI) by inhibiting mitochondrial apoptosis pathway or neuroinflammation in the rat brain damage model [80]. In addition, Sevoflurane post-conditioning promotes autophagy by activating the PI3K/AKT signaling pathway, thereby attenuating TBI-induced neuronal apoptosis [81]. In the study of spinal cord damage, Melatonin enhances autophagy by regulating the PI3K/AKT signaling pathway and reduces cell apoptosis [17]. In this study, we found that Macamide B pretreatment can activate the PI3K/AKT signaling pathway, enhance the expression level of autophagy, and reduce HIBD-induced apoptosis, After intraperitoneal administration of PI3K inhibitor 3MA, 3-MA significantly inhibited the activation of PI3K/AKT signaling pathway and blocked the formation of autophagosomes, resulting in a significant decrease in LC3B and Beclin1 expression levels, and a significant increase in p62 expression levels, Thereby aggravating the apoptosis induced by HIBD (Fig. 14). We confirmed that macamide B pretreatment might regulate autophagy through PI3K/AKT signaling pathway to reduce apoptosis induced by HIBD.

In summary, macamide B pretreatment can effectively treat or prevent HIBD in newborn mice, and its effect may be achieved by regulating autophagy through the PI3K/AKT signaling pathway, thereby reducing HIBD-induced apoptosis. Macamide B may be a potential drug candidate for effective prevention and treatment of neonatal HIBD.

\section{Declarations}

Author Contributions Li Luo and Mengxia Wang established the animal models and providing technical and writing guidance throughout the process ; Xiaoxia Yang contributed in experimental operations, statistical analysis, and manuscript writing; Qian Zhou assisted in the completion of western blot, behavioral tests, immunofluorescence, etc.; Yanxian Bai, Jing Liu, Junhua Yang, and Guoying Li provided technical guidance. All authors read and approved the final manuscript.

Funding This project was financially supported by the National Natural Science Foundation of China for Youth (grant no. 81901524), the Natural Science Foundation of Guangdong Province (2021A1515011525, 2018A030313579), the Guangdong Medical Science and Technology Research Fund (A2020252).

Consent to participate Not applicable to this study

Consent for Publication All authors approve the manuscript for publication

Data and materials availability All data and materials are available on request from authors.

Acknowledgements The authors acknowledge the technical support from Guangdong Pharmaceutical University

Conflict of interest The authors declare that they have no conflict of interest. 
Ethical Approval All applicable international, national, and/or institutional guidelines for the care and use of animals were followed.

\section{References}

1. Gamdzyk M, Doycheva DM, Araujo C, Ocak U, Luo Y, Tang J, Zhang JH (2020) cGAS/STING Pathway Activation Contributes to Delayed Neurodegeneration in Neonatal Hypoxia-Ischemia Rat Model: Possible Involvement of LINE-1. Mol Neurobiol 57(6):2600-2619. https://doi.org/10.1007/s12035020-01904-7

2. Douglas-Escobar M, Weiss MD (2015) Hypoxic-ischemic encephalopathy: a review for the clinician. JAMA Pediatr 169(4):397-403. https://doi.org/10.1001/jamapediatrics.2014.3269

3. Arteni NS, Salgueiro J, Torres I, Achaval M, Netto CA (2003) Neonatal cerebral hypoxia-ischemia causes lateralized memory impairments in the adult rat. Brain Res 973 (2):171178. https://doi.org/10.1016/s0006-8993(03)02436-3

4. Kurinczuk JJ, White-Koning M, Badawi N (2010) Epidemiology of neonatal encephalopathy and hypoxic-ischaemic encephalopathy. Early Hum Dev 86(6):329338. https://doi.org/10.1016/j.earlhumdev.2010.05.010

5. Uria-Avellanal C, Robertson $\mathrm{NJ}(2014) \mathrm{Na}^{+} / \mathrm{H}^{+}$exchangers and intracellular $\mathrm{pH}$ in perinatal brain injury. Transl Stroke Res 5(1):79-98. https://doi.org/10.1007/s12975-013-0322-x

6. Leon J (1963) The "Maca" (Lepidium meyenii), A little known food plant of Peru. Econ Bot 18(2):122127. https://doi.org/10.1007/BF02862707

7. Wang LW, Liang J, Wang XD, Yuan XF, Zhao B, Yang YW (2012) High efficient antioxidant activity of extracts from Lepidium meyenii Walp. Asian Journal of Chemistry 24(10):47954798. https://doi.org/10.1007/s12633-012-9134-y

8. Sandovala M, Okuhama NN, Angeles FM, Melchor VV, Condezo LA, Lao J, Miller MJS (2002) Antioxidant activity of the cruciferous vegetable Maca (Lepidium meyenii). Food Chemistry 79(2):207-213. https://doi.org/10.1016/S0308-8146(02)00133-4

9. Lewis S, Pino-Figueroa A (2013) Neuroprotective effects of Maca extract and macamides against amyloid $\beta$ peptide induced neurotoxicity in B-35 neuroblastoma cells. The FASEB Journal 27:622.19.27(S1):662.619-662.619. https://doi.org/10.1096/fasebj.27.1_supplement.662.19

10. Hajdu Z, Nicolussi S, Rau M, Lorántfy L, Forgo P, Hohmann J, Csupor D, Gertsch J (2014) Identification of endocannabinoid system-modulating $\mathrm{N}$-alkylamides from Heliopsis helianthoides var. scabra and Lepidium meyenii. J Nat Prod 77(7):1663-1669. https://doi.org/10.1021/np500292g

11. Alasmari M, Böhlke M, Kelley C, Maher T, Pino-Figueroa A (2019) Inhibition of Fatty Acid Amide Hydrolase (FAAH) by Macamides. Molecular neurobiology 56 (3):1770-

1781. https://doi.org/10.1007/s12035-018-1115-8

12. Ye YQ, Ma ZH, Yang QF, Sun YQ, Zhang RQ, Wu RF, Ren X, Mu LJ, Jiang ZY, Zhou M (2019) Isolation and synthesis of a new benzylated alkamide from the roots of Lepidium meyenii. Nat Prod Res 
33(19):2731-2737. https://doi.org/10.1080/14786419.2018.1499633

13. Carlsson Y, Schwendimann L, Vontell R, Rousset Cl, Wang X, Lebon S, Charriaut-Marlangue C, Supramaniam V, Hagberg H, Gressens P, Jacotot E (2011) Genetic inhibition of Caspase-2 reduces hypoxic-ischemic and excitotoxic neonatal brain injury. Ann Neurol 70(5):781789. https://doi.org/10.1002/ana.22431

14. Cai CC, Zhu JH, Ye LX, Dai YY, Fang MC, Hu YY, Pan SL, Chen S, Li PJ, Fu XQ, Lin ZL (2019) Glycine Protects against Hypoxic-Ischemic Brain Injury by Regulating Mitochondria-Mediated Autophagy via the AMPK Pathway. Oxid Med Cell Longev 2019:4248529. https://doi.org/10.1155/2019/4248529

15. Shi X, Xu L, Doycheva DM, Tang J, Yan M, Zhang JH (2017) Sestrin2, as a negative feedback regulator of $\mathrm{mTOR}$, provides neuroprotection by activation AMPK phosphorylation in neonatal hypoxic-ischemic encephalopathy in rat pups. J Cereb Blood Flow Metab 37(4):14471460. https://doi.org/10.1177/0271678X16656201

16. Wasan H, Singh D, Joshi B, Sharma U, Dinda AK, Reeta KH (2021) Post Stroke Safinamide Treatment Attenuates Neurological Damage by Modulating Autophagy and Apoptosis in Experimental Model of Stroke in Rats. Mol Neurobiol Aug 28. https://doi.org/10.1007/s12035-021-02523-6

17. Li Y, Guo Y, Fan Y, Tian H, Li K, Mei X (2019) Melatonin Enhances Autophagy and Reduces Apoptosis to Promote Locomotor Recovery in Spinal Cord Injury via the PI3K/AKT/mTOR Signaling Pathway. Neurochem Res 44(8):2007-2019. https://doi.org/10.1007/s11064-019-02838-w

18. Lipinski MM, Zheng B, Lu T, Yan Z, Py BF, Ng A, Xavier RJ, Li C, Yankner BA, Scherzer CR, Yuan J (2010) Genome-wide analysis reveals mechanisms modulating autophagy in normal brain ageing and in Alzheimer's disease. Proc Natl Acad Sci U S A 107(32):1416414169. https://doi.org/10.1073/pnas. 1009485107

19. Kotoda M, Furukawa H, Miyamoto T, Korai M, Shikata F, Kuwabara A, Xiong X, Rutledge C, Giffard RG, Hashimoto T (2018) Role of Myeloid Lineage Cell Autophagy in Ischemic Brain Injury. Stroke 49(6):1488-1495. https://doi.org/10.1161/strokeaha.117.018637

20. Feng D, Wang B, Wang L, Abraham N, Tao K, Huang L, Shi W, Dong Y, Qu Y (2017) Pre-ischemia melatonin treatment alleviated acute neuronal injury after ischemic stroke by inhibiting endoplasmic reticulum stress-dependent autophagy via PERK and IRE1 signalings. J Pineal Res 62(3). https://doi.org/10.1111/jpi.12395

21. Qin AP, Liu CF, Qin YY, Hong LZ, Xu M, Yang L, Liu J, Qin ZH, Zhang HL (2010) Autophagy was activated in injured astrocytes and mildly decreased cell survival following glucose and oxygen deprivation and focal cerebral ischemia. Autophagy 6(6):738-

753. https://doi.org/10.4161/auto.6.6.12573

22. Wang MM, Zhang M, Feng YS, Xing Y, Tan ZX, Li WB, Dong F, Zhang F (2020) Electroacupuncture Inhibits Neuronal Autophagy and Apoptosis via the PI3K/AKT Pathway Following Ischemic Stroke. Frontiers in cellular neuroscience 14:134. https://doi.org/10.3389/fncel.2020.00134

23. Guo SS, Gao XF, Gu YR, Wan ZX, Lu AM, Qin ZH, Luo L (2016) Preservation of Cognitive Function by Lepidium meyenii (Maca) Is Associated with Improvement of Mitochondrial Activity and 
Upregulation of Autophagy-Related Proteins in Middle-Aged Mouse Cortex. Evidence-based complementary and alternative medicine: eCAM

2016:4394261. https://doi.org/10.1155/2016/4394261

24. Liu F, Wang Y, Yao W, Xue Y, Zhou J, Liu Z (2019) Geniposide attenuates neonatal mouse brain injury after hypoxic-ischemia involving the activation of PI3K/Akt signaling pathway. J Chem Neuroanat 102:101687. https://doi.org/10.1016/j.jchemneu.2019.101687

25. Tian Z, Tang C, Wang Z (2019) Neuroprotective effect of ginkgetin in experimental cerebral ischemia/reperfusion via apoptosis inhibition and PI3K/Akt/mTOR signaling pathway activation. J Cell Biochem 120(10):18487-18495. https://doi.org/10.1002/jcb.29169

26. Zhou Z, Xu N, Matei N, McBride DW, Ding Y, Liang H, Tang J, Zhang JH (2021) Sodium butyrate attenuated neuronal apoptosis via GPR41/GßY/PI3K/Akt pathway after MCAO in rats. J Cereb Blood Flow Metab 41(2):267-281. https://doi.org/10.1177/0271678x20910533

27. Jin Y, Shang Y, Zhang D, An J, Pan D (2019) Hexabromocyclododecanes promoted autophagy through the PI3K/Akt/mTOR pathway in L02 cells. J Environ Manage 244:77-

82. https://doi.org/10.1016/j.jenvman.2019.05.031

28. Guo Y, Wang J, Wang Z, Yang Y, Wang X, Duan Q (2010) Melatonin protects N2a against ischemia/reperfusion injury through autophagy enhancement. J Huazhong Univ Sci Technolog Med Sci 30(1):1-7. https://doi.org/10.1007/s11596-010-0101-9

29. Zhang X, Yuan Y, Jiang L, Zhang J, Gao J, Shen Z, Zheng Y, Deng T, Yan H, Li W, Hou WW, Lu J, Shen Y, Dai H, Hu WW, Zhang Z, Chen Z (2014) Endoplasmic reticulum stress induced by tunicamycin and thapsigargin protects against transient ischemic brain injury: Involvement of PARK2-dependent mitophagy. Autophagy 10(10):1801-1813. https://doi.org/10.4161/auto.32136

30. Zhang RR, Meng NN, Liu C, Li KL, Wang MX, Lv ZB, Chen SY, Guo X, Wang XK, Wang Q, Sun JY (2020) PDB-1 from Potentilla discolor Bunge induces apoptosis and autophagy by downregulating the PI3K/Akt/mTOR signaling pathway in A549 cells. Biomed Pharmacother 129:110378. https://doi.org/10.1016/j.biopha.2020.110378

31. Xu N, Zhang Y, Doycheva DM, Ding Y, Zhang Y, Tang J, Guo H, Zhang JH (2018) Adiponectin attenuates neuronal apoptosis induced by hypoxia-ischemia via the activation of AdipoR1/APPL1/LKB1/AMPK pathway in neonatal rats. Neuropharmacology 133ø415428. https://doi.org/10.1016/j.neuropharm.2018.02.024

32. Rice JE, Vannucci RC, Brierley JB (1981) The influence of immaturity on hypoxic-ischemic brain damage in the rat. Ann Neurol 9(2):131-141. https://doi.org/10.1002/ana.410090206

33. Xu B, Xiao AJ, Chen W, Turlova E, Liu R, Barszczyk A, Sun CLF, Liu L, Tymianski M, Feng ZP, Sun HS (2016) Neuroprotective Effects of a PSD-95 Inhibitor in Neonatal Hypoxic-Ischemic Brain Injury. Mol Neurobiol 53(9):5962-5970. https://doi.org/10.1007/s12035-015-9488-4

34. Tymianski, M (2011) Emerging mechanisms of disrupted cellular signaling in brain ischemia. Nat Neurosci 14(11):1369-1373. https://doi.org/10.1038/nn.2951 
35. Bo Q, Shen M, Xiao M, Liang J, Zhai Y, Zhu H, Jiang M, Wang F, Luo X, Sun X (2020) 3-Methyladenine Alleviates Experimental Subretinal Fibrosis by Inhibiting Macrophages and M2 Polarization Through the PI3K/Akt Pathway. J Ocul Pharmacol Ther 36(8):618-

628. https://doi.org/10.1089/jop.2019.0112

36. Millar LJ, Shi L, Hoerder-Suabedissen A, Molnar Z (2017) Neonatal Hypoxia Ischaemia: Mechanisms, Models, and Therapeutic Challenges. Front Cell Neurosci

11:78. https://doi.org/10.3389/fncel.2017.00078

37. Herz J, Köster C, Crasmöller M, Abberger H, Hansen W, Felderhoff-Müser U, Bendix I (2018) Peripheral T cell depletion by FTY720 exacerbates hypoxic-ischemic brain injury in neonatal mice. Front Immunol 9:1696. https://doi.org/10.3389/fimmu.2018.01696

38. Shankaran S, Laptook AR, pappas A, McDonald SA, Das A, Tyson JE, Poindexter BB, Schibler K, Bell EF, Heyne RJ, Pedroza C, Bara R, Van Meurs KP, Huitema CMP, Grisby C, Devaskar U, Ehrenkranz RA, Harmon HM, Chalak LF, DeMauro SB, Garg M, Hartley-McAndrew ME, Khan AM, Walsh MC, Ambalavanan N, Brumbaugh JE, Watterberg KL, Shepherd EG区 Hamrick SEG, Barks J, Cotten CM, Kilbride HW, Higgins RD, Eunice Kennedy Shriver National Institute of Child Health and Human Development Neonatal Research Network (2017) Effect of depth and duration of cooling on death or disability at age 18 months among neonates with hypoxic-ischemic encephalopathy: a randomized clinical trial. JAMA vol 318(1):57-67. https://doi.org/10.1001/jama.2017.7218

39. Schabitz WR, Fisher M (2006) Perspectives on neuroprotective stroke therapy. Biochem Soc Trans 34:1271-1276. https://doi.org/10.1042/BST0341271

40. Mehta SL, Manhas N, Raghubir R (2007) Molecular targets in cerebral ischemia for developing novel therapeutics. Brain Res Rev 54(1):34-66. https://doi.org/10.1016/j.brainresrev.2006.11.003

41. Choi EH, Kang JI, Cho JY, Lee SH, Kim TS, Yeo IH, Chun HS (2012) Supplementation of standardized lipid-soluble extract from maca (Lepidium meyenii) increases swimming endurance capacity in rats. J Funct Foods 4(2):568-573. https://doi.org/10.1016/j.jff.2012.03

42. Zhang Y, Yu L, Ao M, Jin W (2006) Effect of ethanol extract of Lepidium meyenii Walp. on osteoporosis in ovariectomized rat. J Ethnopharmacol 105(1):274-

279. https://doi.org/10.1016/j.jep.2005.12.013

43. Valentová K, Ulrichová J (2003) Smallanthus sonchifolius and Lepidium meyenii - prospective Andean crops for the prevention of chronic diseases. Biomed Pap Med Fac Univ Palacky Olomouc Czech Repub 147(2):119-130.

44. Zheng BL, He K, Kim CH, Rogers L, Shao Y, Huang ZY, Lu Y, Yan SJ, Qien LC, Zheng QY (2000) Effect of a lipidoc extract from lepidium meyenii on sexual behavior in mice and rats. Urology 55(4):598602. https://doi.org/10.1016/s0090-4295(99)00549-x

45. Martorana A, Mori F, Esposito Z, Kusayanagi H, Monteleone F, Codecà C, Sancesario G, Bernardi G, Koch G (2009) Dopamine Modulates Cholinergic Cortical Excitability in Alzheimer's Diseasee Patients. Neuropsychopharmacology 34(10):2323-2328. https://doi.org/10.1038/npp.2009.60 
46. Zhou Y, Li P, Brantner A, Wang H, Shu X, Yang J, Si N, Han L, Zhao H, Bian Baolin (2017) Chemical Profiling analysis of Maca using UHPL-ESI-Orbitrap MS coupled with UHPLC-ESI-QqQ MS and the neuroprotective study on its active ingredients. Sci Rep 7:44660. https://doi.org/10.1038/srep44660

47. Zhao YJ, Nai Y, Li SY, Zheng YH (2018) Retigabine protects the blood-brain barrier by regulating tight junctions between cerebral vascular endothelial cells in cerebral ischemia-reperfusion rats. Eur Rev Med Pharmacol Sci 22(23):8509-8518. https://doi.org/10.26355/eurrev_201812_16552

48. Buckholtz NS (1980) Neurobiology of tetrahydro-beta-carbolines. Life Sci 27(11):893-903. https://doi.org/10.1016/0024-3205(80)90098-3

49. Gonzales GF, Gonzales-Castañeda C (2008) The Methyltetrahydro-\{beta\}-carbolines in maca (Lepidium meyenii). Evid Based Complement Alternat Med 6(3):315316. https://doi.org/10.1093/ecam/nen041

50. Herraiz, T (2000) Tetrahydro-beta-carbolines, potential neuroactive alkaloids, in chocolate and cocoa. J Agric Food Chem 48(10):4900-4904. https://doi.org/10.1021/jf000508।

51. Liu XM, Feng Y, Li AM (2015) Efect of G-CSF and TPO on HIBD in neonatal rats. Asian Pac J Trop Med 8(2):132-136. https://doi.org/10.1016/s1995-7645(14)60303-5

52. Johnston MV, Ferriero DM,Vannucci SJ, Hagberg H (2005) Models of cerebral palsy: which ones are best? J.Child Neurol 20(12):984-987. https://doi.org/10.1177/08830738050200121001

53. Towfghi J, Mauger D, Vannucci RC, Vannucci SJ (1997) Infuence of age on the cerebral lesions in an immature rat model of cerebral hypoxia-ischemia: a light microscopic study. Brain Res Dev Brain Res 100(2):149-160. https://doi.org/10.1016/s0165-3806(97)00036-9

54. Zheng Z, Zhang L, Qu Y, Xiao G, Li S, Bao S, Lu QR, Mu D (2018) Mesenchymal stem cells protect against hypoxia ischemia brain damage by enhancing autophagy through BDNF/mTOR signaling pathway. Stem Cells 36(7):1109-1121. https://doi.org/10.1002/stem.2808

55. Banasiak KJ, Xia Y, Haddad GG (2000) Mechanisms underlying hypoxia-induced neuronal apoptosis. Prog Neurobiol. 62(3):215-249. https://doi.org/10.1016/s0301-0082(00)00011-3

56. Sidhu RS, Tuor UI, Del Bigio MR (1997) Nuclear condensation and fragmentation following cerebral hypoxia-ischemia occurs more frequently in immature than older rats. Neurosci Lett 223(2):129132. https://doi.org/10.1016/s0304-3940(97)13426-7

57. Northington FJ, Graham EM, Martin LJ (2005) Apoptosis in perinatal hypoxic-is-chemic brain injury: How important is it and should it be inhibited? Brain Res BrainRes Rev 50(2):244257. https://doi.org/10.1016/j.brainresrev.2005.07.003

58. Zhang X, Kan H, Liu Y, Ding W (2021) Plumbagin induces Ishikawa cell cycle arrest, autophagy, and apoptosis via the PI3K/Akt signaling pathway in endometrial cancer. Food and chemical toxicology Food Chem Toxicol 148:111957. https://doi.org/10.1016/j.fct.2020.111957

59. Gill R, Soriano M, Blomgren K, Hagberg H, Wybrecht R, Miss MT, Hoefer S, Adam G, Niederhauser O, Kemp JA, Loetscher H (2002) Role of caspase-3 activation in cerebral ischemia-induced neurodegeneration in adult and neonatal brain. J Cereb Blood Flow Metab 22(4):420430. https://doi.org/10.1097/00004647-200204000-00006 
60. Haylor JL, Harris KPG, Nicholson ML, Waller HL, Huang Q, Yang B (2011) Atorvastatin improving renal ischemia reperfusion injury via direct inhibition of active caspase-3 in rats. Exp Biol Med (Maywood) 236(6):755-763. https://doi.org/10.1258/ebm.2011.010350

61. Zhu HC, Gao XQ, Xing Y, Sun SG, Li HG, Wang YF (2004) Inhibition of Caspase 3 activation and apoptosis is involved in 3-nitropropionic acid-induced ischemic tolerance to transient focal cerebral ischemia in rats. J Mol Neurosci 24(2):299-305. https://doi.org/10.1385/jmn:24:2:299

62. Zhang C, Huang C, Yang P, Li C, Li M (2021) ROS to suppress the PI3K/Akt/mTOR signaling pathway. Cell Signal 78:109841. https://doi.org/10.1016/j.cellsig.2020.109841

63. Zhou J, Jiang YY, Chen H, Wu YC, Zhang L (2020) Tanshinone I attenuates the malignant biological properties of ovarian cancer by inducing apoptosis and autophagy via the inactivation of PI3K/AKT/mTOR pathway. Cell Prolif 53(2):e12739. https://doi.org/10.1111/cpr.12739

64. Li TF, Ma J, Han XW, Jia YX, Yuan HF, Shui SF, Guo D, Yan L (2019) Chrysin ameliorates cerebral ischemia/reperfusion (I/R) injury in rats by regulating the PI3K/Akt/mTOR pathway. Neurochem Int 129:104496. https://doi.org/10.1016/j.neuint.2019.104496

65. Huang TJ, Ren JJ, Zhang QQ, Kong YY, Zhang HY, Guo XH, Fan HQ, Liu LX (2019) IGFBPrP1 accelerates autophagy and activation of hepatic stellate cells via mutual regulation between $\mathrm{H} 19$ and PI3K/AKT/mTOR pathway. Biomed Pharmacother

116:109034. https://doi.org/10.1016/j.biopha.2019.109034

66. Li A, Li X, Chen X, Zeng C, Wang Z, Li Z, Chen J (2020) NUPR1 Silencing Induces AutophagyMediated Apoptosis in Multiple Myeloma Cells Through the PI3K/AKT/mTOR Pathway. DNA Cell Biol 39(3):368-378. https://doi.org/10.1089/dna.2019.5196

67. Guo H, Zhao L, Wang B, Li X, Bai H, Liu H, Yue L, Guo W, Bian Z, Gao L, Feng D, Qu Y (2018) Remote limb ischemic postconditioning protects against cerebral ischemia-reperfusion injury by activating AMPK-dependent autophagy. Brain Res Bull 139:105-

113. https://doi.org/10.1016/j.brainresbull.2018.02.013

68. Zhang Y, Zhang Y, Jin XF, Zhou XH, Dong XH, Yu WT, Gao WJ (2019) The Role of Astragaloside IV against Cerebral Ischemia/Reperfusion Injury: Suppression of Apoptosis via Promotion of P62-LC3Autophagy. Molecules (Basel, Switzerland) 24(9). https://doi.org/10.3390/molecules24091838

69. Zhang F, Dong H, Lv T, Jin K, Jin Y, Zhang X, Jiang J (2018) Moderate hypothermia inhibits microglial activation after traumatic brain injury by modulating autophagy/apoptosis and the MyD88dependent TLR4 signaling pathway. J Neuroinflammation 15(1):273. https://doi.org/10.1186/s12974-018-1315-1

70. Sun Y, Zhang T, Zhang Y, Li J, Jin, L, Shi N, Liu K, Sun X (2018b). Ischemic postconditioning alleviates cerebral ischemia-reperfusion injury through activating autophagy during early reperfusion in rats. Neurochem Res 43(9):1826-1840. https://doi.org/10.1007/s11064-018-2599-3

71. Feng J, Chen X, Guan B, Li C, Qiu J, Shen J (2018) Inhibition of Peroxynitrite-Induced Mitophagy Activation Attenuates Cerebral Ischemia-Reperfusion Injury. Mol Neurobiol 55(8):63696386. https://doi.org/10.1007/s12035-017-0859-x 
72. Sun X, Wang D, Zhang T, Lu X, Duan F, Ju L, Zhuang X, Jiang X (2020) Eugenol Attenuates Cerebral Ischemia-Reperfusion Injury by Enhancing Autophagy via AMPK-mTOR-P70S6K Pathway. Front Pharmacol 11:84. https://doi.org/10.3389/fphar.2020.00084

73. Ye L, Wang X, Cai C, Zeng S, Bai J, Guo K, Fang M, Hu J, Liu H, Zhu L, Liu F, Wang D, Hu Y, Pan S, Li X, Lin L, Lin Z (2019) FGF21 promotes functional recovery after hypoxic-ischemic brain injury in neonatal rats by activating the PI3K/AKT signaling pathway via FGFR1/ß-klotho Exp Neurol 317:3450. https://doi.org/10.1016/j.expneurol.2019.02.013

74. Xian G, Chen W, Gu M, Ye Y, Yang G, Lai W, Xiao Y, Zhao X, Zheng L, Pan B, Kang Y, Zhang Z, Sheng P (2020) Titanium particles induce apoptosis by promoting autophagy in macrophages via the PI3K/Akt signaling pathway. J Biomed Mater Res A 108(9):1792-

1805. https://doi.org/10.1002/jbm.a.36938

75. Zhang W, Liu J, Hu X, Li P, Leak RK, Gao Y, Chen J (2015) n-3 polyunsaturated fatty acids reduce neonatal Hypoxic/Ischemic brain injury by promoting phosphatidylserine formation and AKT signaling. Stroke 46(10):2943-2950. https://doi.org/10.1161/STROKEAHA.115.010815

76. Tu L, Wang Y, Chen D, Xiang P, Shen J, Li Y, Wang S (2018) Protective effects of Notoginsenoside R1 via regulation of the PI3K-AKT-mTOR/JNK pathway in neonatal cerebral hypoxic-ischemic brain injury. Neurochem Res 43(6):1210-1226. https://doi.org/10.1007/s11064-018-2538-3

77. Rychahou PG, Jackson LN, Silva SR, Rajaraman S, Evers BM (2006) Targeted molecular therapy of the PI3K pathway therapeutic significance of PI3K subunit targeting in colorectal carcinoma. Ann Surg 243(6):833-844. https://doi.org/10.1097/01.sla.0000220040.66012.a9

78. Cubero-Gómez JM, Guerrero Márquez FJ, La-Llera DD, Fernández-Quero M, Guisado-Rasco A, VillaGil-Ortega M (2017) Severe thrombocytopenia induced by iodinated contrast after coronary an giography: The use of gadolinium contrast and intravascular ultra sound as an alternative to guide percutaneous coronary intervention. Rev Port Cardiol 36(1):61.e1-

61.e4. https://doi.org/10.1016/j.repc.2016.04.014

79. Tan XH, Zhang KK, Xu JT, Qu D, Chen LJ, Li JH, Wang Q, Wang HJ, Xie XL (2020) Luteolin alleviates methamphetamine-induced neurotoxicity by suppressing PI3K/Akt pathway-modulated apoptosis and autophagy in rats. Food Chem Toxicol:an international journal published for the British Industrial Biological Research Association 137:111179. https://doi.org/10.1016/j.fct.2020.111179

80. Lin C, Chao H, Li Z, Xu X, Liu Y, Hou L, Liu N, Ji J (2016) Melatonin attenuates traumatic brain injuryinduced inflammation: a possible role for mitophagy. Journal of pineal research 61(2):177186. https://doi.org/10.1111/jpi.12337

81. He H, Liu W, Zhou Y, Liu Y, Weng P, Li Y, Fu H (2018) Sevoflurane post-conditioning attenuates traumatic brain injury-induced neuronal apoptosis by promoting autophagy via the PI3K/AKT signaling pathway. Drug Des Devel Ther 12:629-638. https://doi.org/10.2147/dddt.S158313

\section{Figures}


a

Chemical structure of macamide B<smiles>CCCCCCCCCCCCCCCC(=O)NCc1ccccc1</smiles>

C

Timelines of study

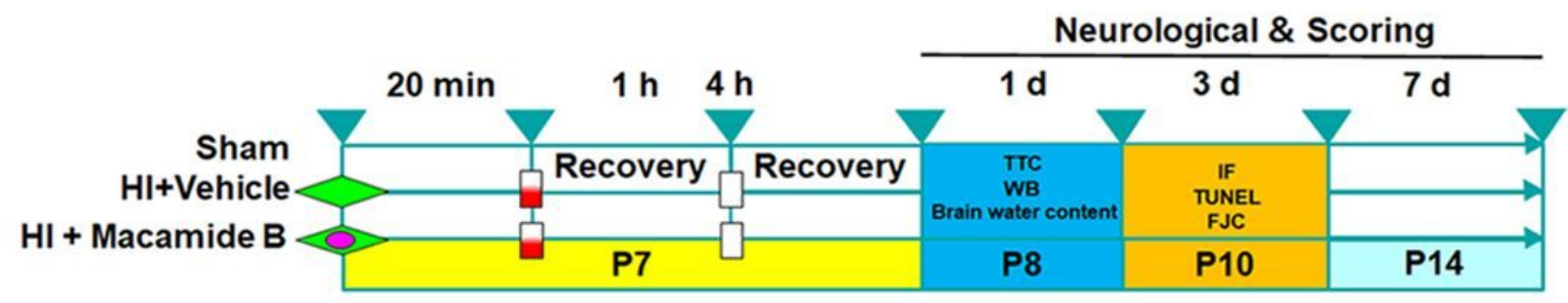

PBS (ip)

Macamide B (ip, $60 \mathrm{mg} / \mathrm{kg}$ ) b

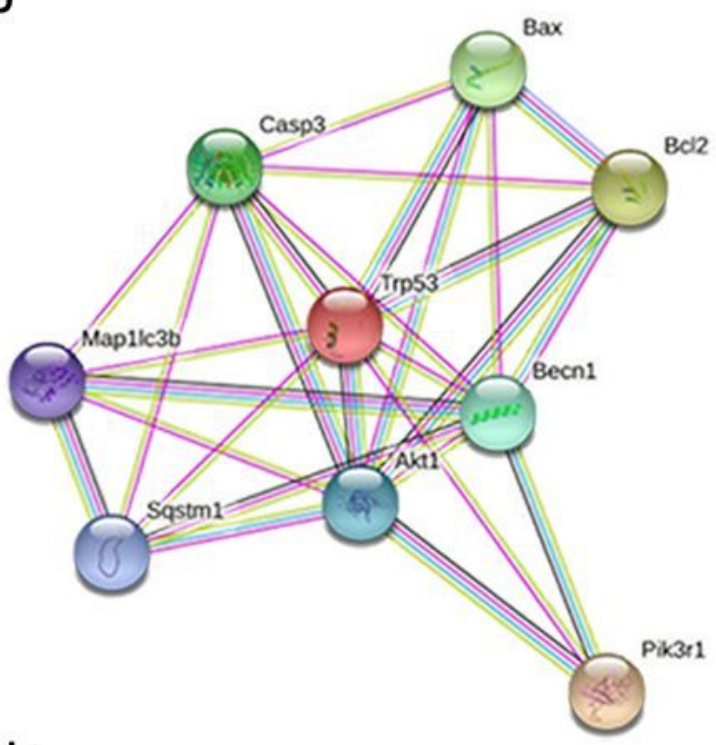

Neurological \& Scoring 
Effect of macamide B pretreatment on HIBD in newborn mice. a Representative pictures of coronal brain sections stained with TTC $24 \mathrm{~h}$ after HIBD. b Quantitative analysis results of cerebral infarct volume in pups. Compared with pretreatment with the vehicle, pretreatment with the medium dose $(60 \mathrm{mg} / \mathrm{kg})$ of macamide B significantly reduced the infarct volume. c Quantitative analysis results of brain water content. The water content of the ipsilateral cerebral hemisphere of the vehicle group was significantly higher than that of the sham group, and the brain water content of the macamide B group was significantly lower than that of the vehicle group. ${ }^{*} p<0.05$ vs. sham group; ${ }^{* \star} p<0.001$ vs. sham group; $\star \star \star p=0.0003$ vs. sham group; $* \star \star \star p<0.0001$ vs. sham group; $\# p<0.05$ vs. vehicle group; $\# \# p=0.001$ vs. vehicle group; \#\#\#\#< 0.0001 vs. vehicle group; @p $<0.01$ vs. macamide B group. $n=6$ for each group.

a

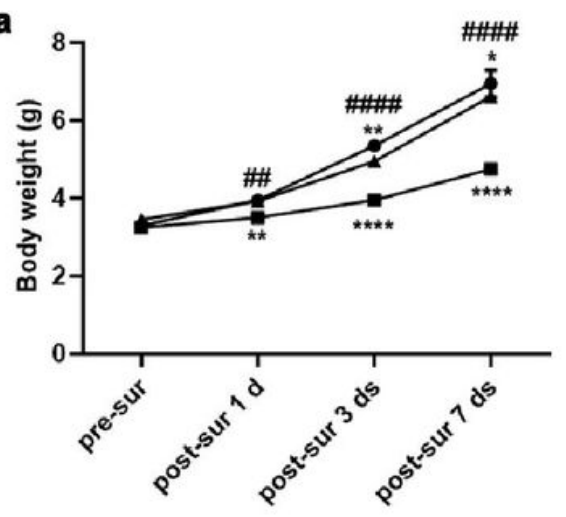

c

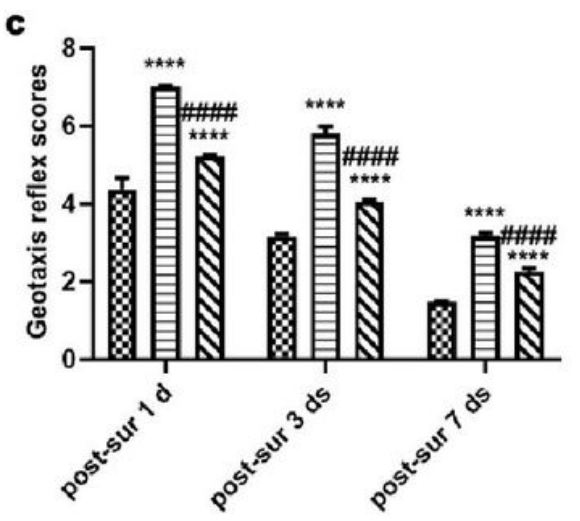

- Sham

- HI+Vehicle

- HI+Macamide B

m Sham

ㅁ HI+Vehicle

WI+Macamide B b

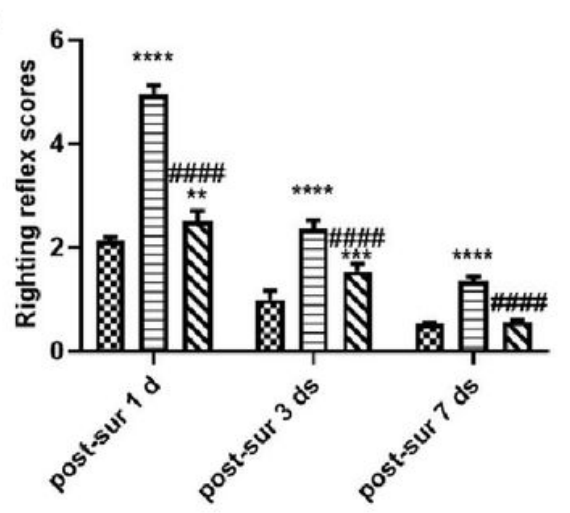

d

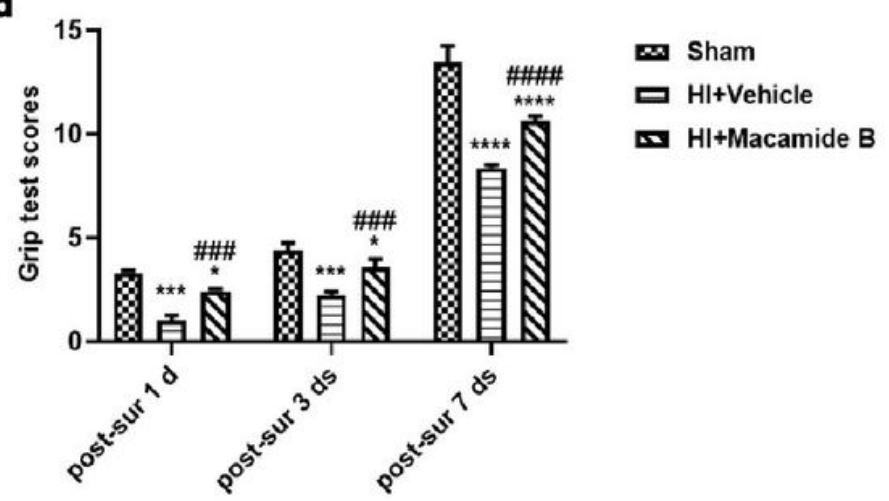

\section{Figure 3}

Macamide B pretreatment improved the neurological function and reversed body weight loss of mice after HIBD. a Mice in the macamide B group had significantly higher body weights than mice in the vehicle group at 1,3 , and 7 days post-surgery. The macamide B pretreatment group exhibited notably improved neurobehavioral outcomes of the $b$ righting reflex, $c$ negative geotaxis, and $d$ grip test at 1,3 , and 7 days postsurgery compared to the vehicle group. ( $\left.{ }^{\star} \mathrm{p}<0.05,{ }^{\star \star} \mathrm{p}<0.01\right) . \mathrm{n}=8$ for each group. 
a

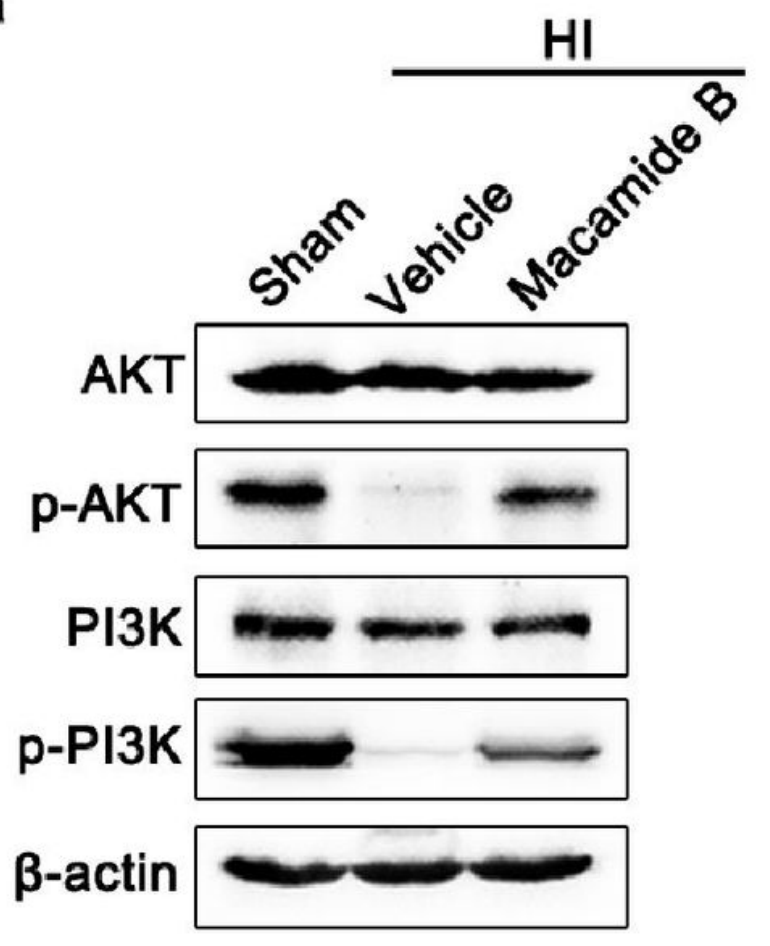

b

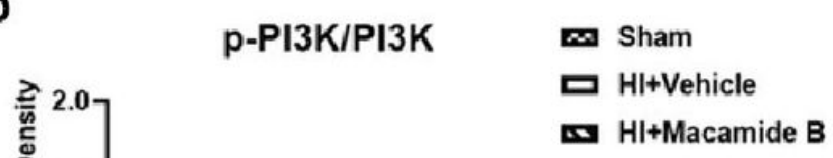

Figure 4

The effect of macamide B pretreatment on PI3K-AKT signaling pathway in HIBD neonatal mice. a Representative western blots and quantification data of $\mathrm{b}$ p-PI3K and $\mathrm{c} p$-Akt protein in the ipsilateral cerebral hemisphere were detected $24 \mathrm{~h}$ after HIBD. Macamide B pretreatment significantly restored $\mathrm{p}$ $\mathrm{PI} 3 \mathrm{~K}$ and p-Akt protein expression in HI brains. ${ }^{* \star \star} \mathrm{p}<0.0001$ vs. sham group; \#\#\#\#p $<0.0001$ vs. vehicle group. $\mathrm{n}=6$ for each group. 


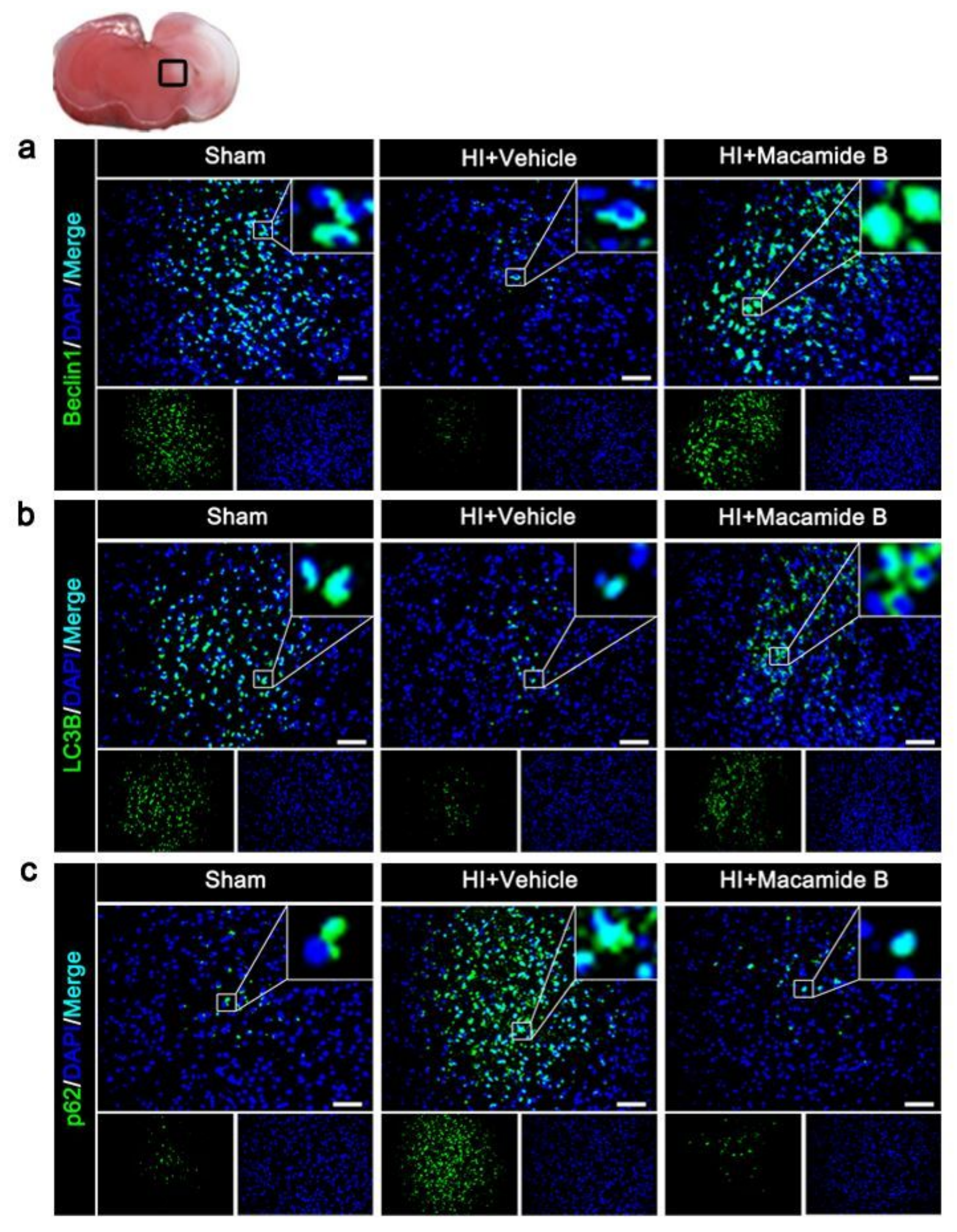

Figure 5

Representative microphotographs of a Beclin1 (green fluorescence), b LC3B (green fluorescence), c p62 (green fluorescence) positive cells in the ipsilateral cortex of HIBD neonatal mice. The blue fluorescence represents DAPI, and merge is the merged image of the target cell and the corresponding nucleus. After HIBD in newborn mice, the number of Beclin1 and LC3B positive cells decreased significantly, and the number of p62 positive cells increased significantly. Macamide B pretreatment significantly improved this 
result. Macamide B significantly increased the number of Beclin1 and LC3B positive cells and inhibited the increase in the number of p62 positive cells. The brain slice in the upper left corner shows the position of immunofluorescence staining (small black box). $n=8$ for each group. Scale bar $=100 \mu \mathrm{m}$.

a

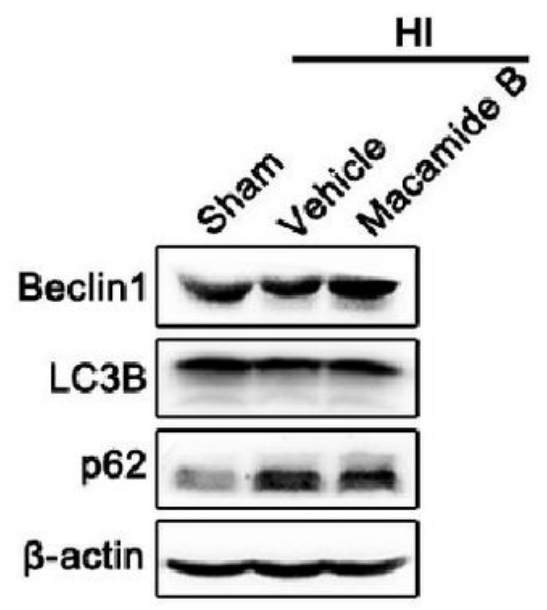

C

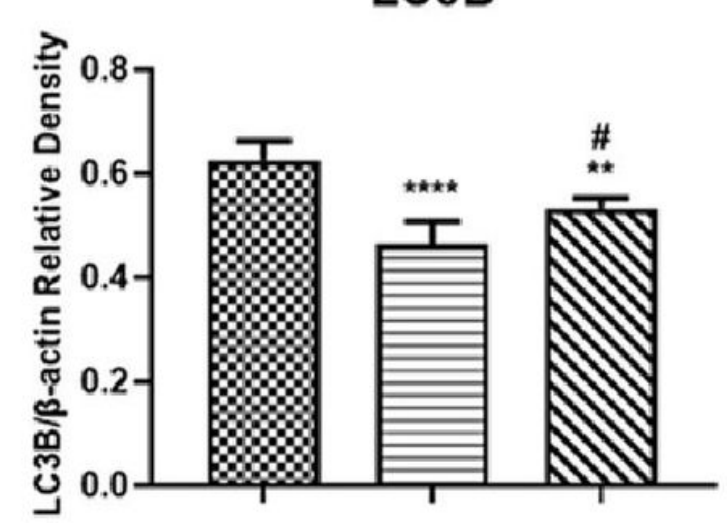

b

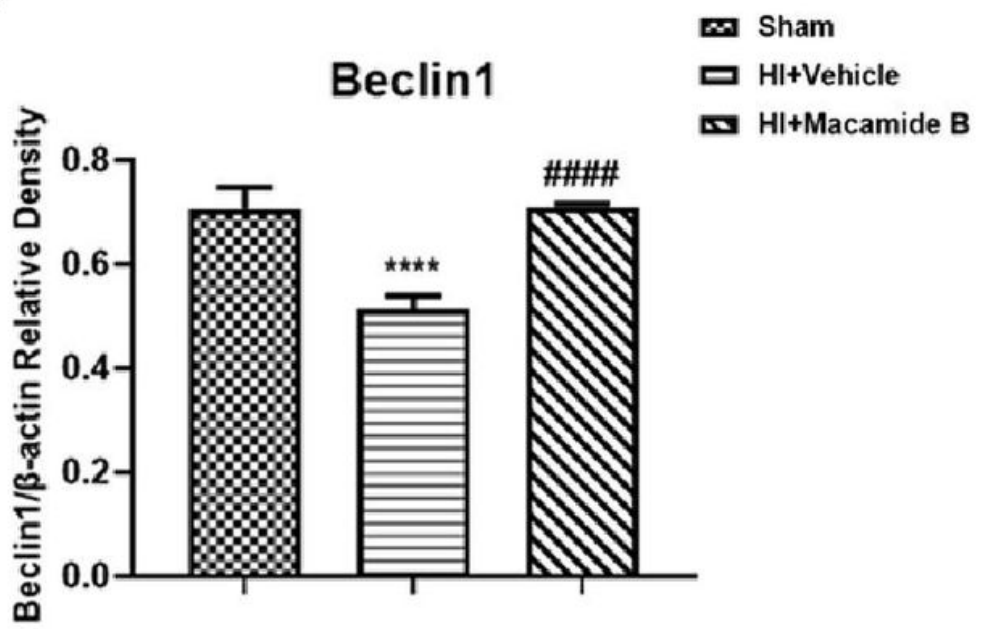

d

p62

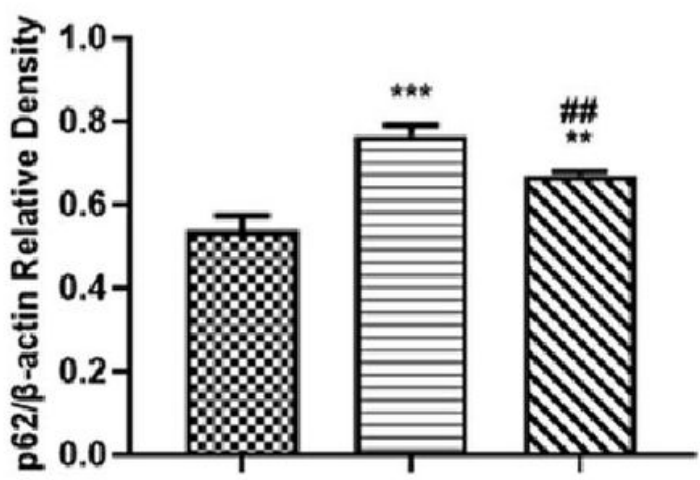

Figure 6

The effect of macamide B pretreatment on autophagy in HIBD neonatal mice. a Representative western blots and quantification data of b Beclin1, c LC3B and d p62 protein in the ipsilateral cerebral hemisphere were detected $24 \mathrm{~h}$ after HIBD. Macamide B pretreatment significantly up-regulates the expression levels of Beclin1 and LC3B in HIBD pups, and down-regulates the expression levels of $p 62 .{ }^{* \star \star \star} p<0.0001$ vs. sham group; ${ }^{\star \star \star} p<0.0001$ vs. sham group; $* \star p<0.01$ vs. sham group; $\# \# \# \# p<0.0001$ vs. vehicle group; $\# p<0.05$ vs. vehicle group; \#\#p<0.01 vs. vehicle group. $n=6$ for each group. 


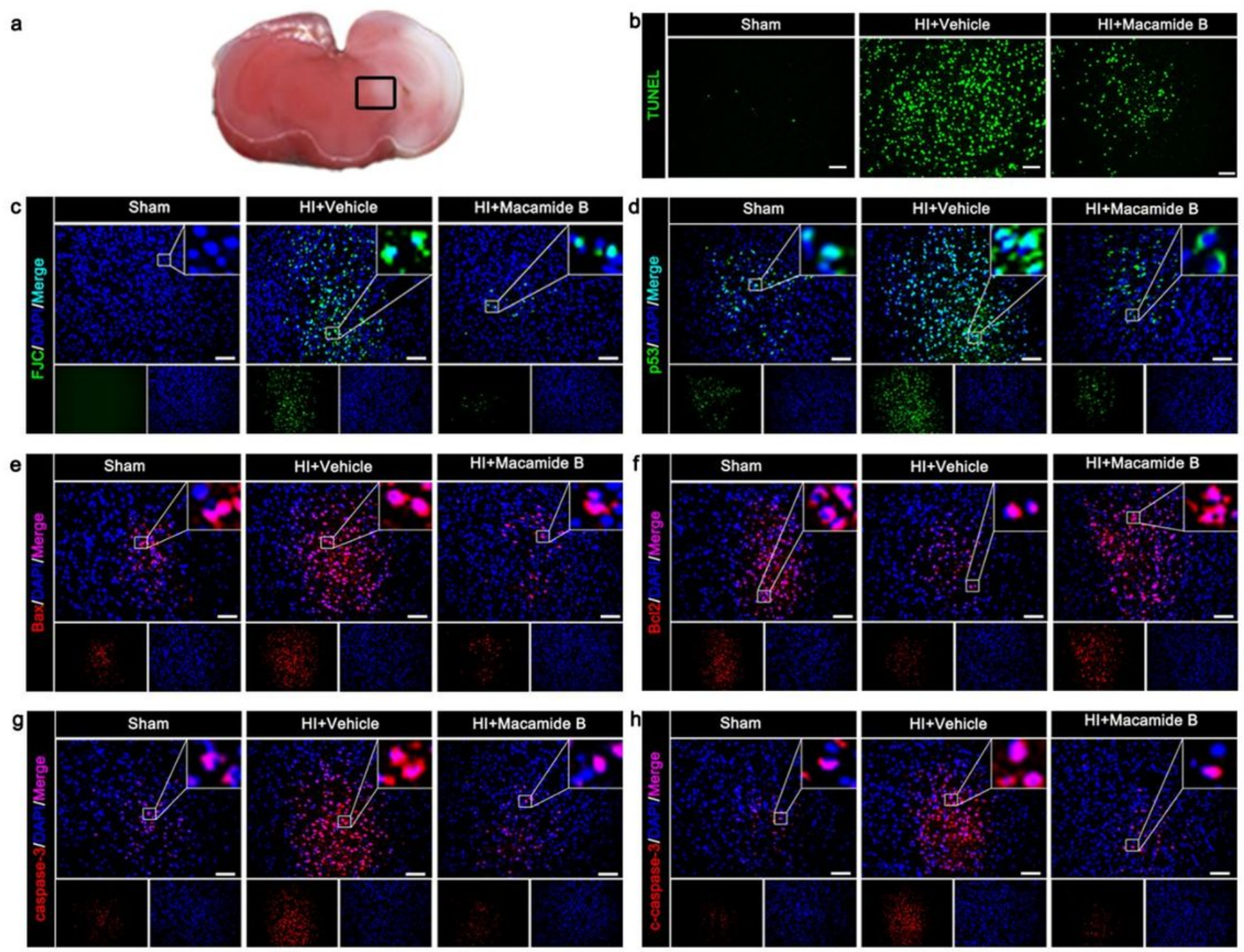

Figure 7

Typical micrographs of b TUNEL-positive cells (green), c FJC-positive neurons (green), and d p53 (green), e Bax (red), f Bcl-2 (red), g caspase-3 (red), and h cleaved caspase-3 (red) positive cells in the ipsilateral cortex of HIBD newborn mice. Blue fluorescence represented DAPI, and merge was the combination of the target cell and the corresponding nucleus. After HIBD in newborn mice, the number of TUNEL positive cells and FJC positive neurons increased significantly. Macamide B pretreatment significantly reversed this result, and significantly reduced the number of TUNEL positive cells and FJC positive neurons, which indicated that macamide B pretreatment could inhibit apoptosis. After neonatal mice HIBD treatment, the number of apoptotic related proteins p53, Bax, caspase-3, and cleaved caspase-3 positive cells was significantly increased, and the number of Bcl-2 positive cells was significantly decreased. Macamide B pretreatment significantly improved this result and played an anti-apoptotic effect on neonatal mice HIBD. i panel indicates the location of staining (small black box). $n=8$ for each group. Scale bar $=100$ $\mu \mathrm{m}$. 
a

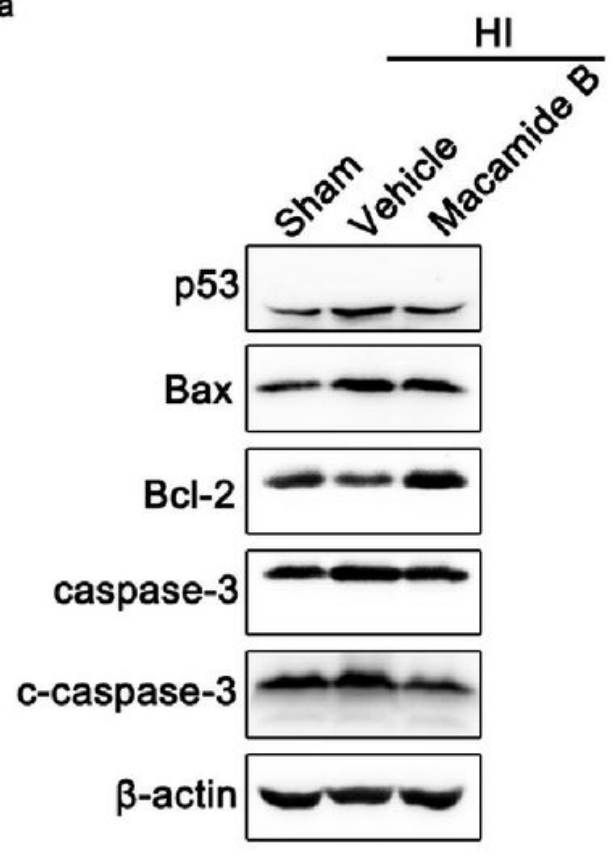

b

p53

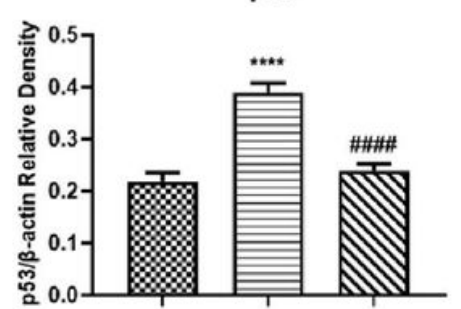

d

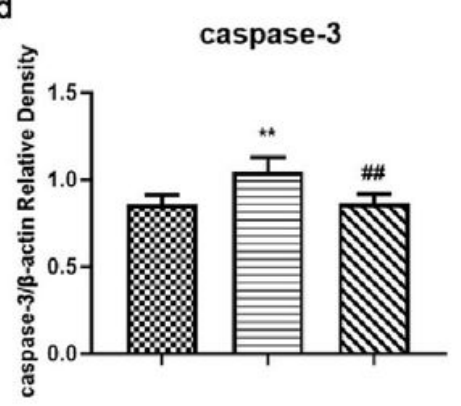

c
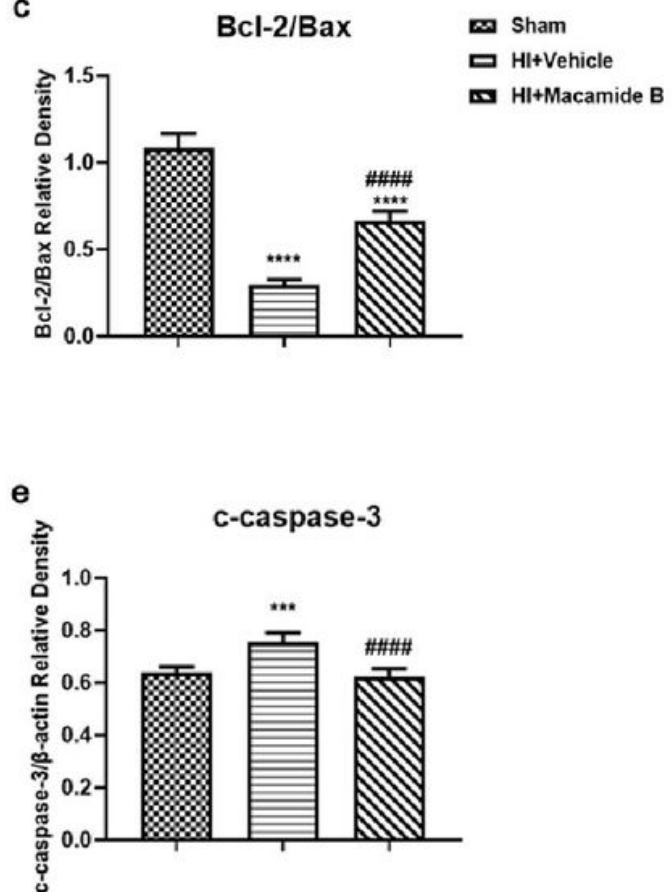

\section{Figure 8}

The effect of macamide B pretreatment on cell apoptosis in HIBD neonatal mice. a Representative western blots and quantification data of b p53, c Bcl-2 / d Bax, e caspase-3, and f cleaved caspase-3 in the ipsilateral cerebral hemisphere were detected $24 \mathrm{~h}$ after HIBD. Macamide B pretreatment significantly down-regulated p53, caspase-3, and cleaved caspase-3 protein expressions and up-regulated the $\mathrm{Bcl} 2 / \mathrm{Bax}$ ratio when compared with the vehicle group. Western blot results showed that macamide $\mathrm{B}$ pretreatment significantly reduced the apoptosis of HIBD neonatal mice. $* \star \star * p<0.0001$ vs. sham group; $\star \star p<0.01$ vs. sham group; $* \star \star p<0.0001$ vs. sham group; \#\#\#\#p $<0.0001$ vs. vehicle group; \#\#p $<0.05$ vs. vehicle group. $\mathrm{n}=6$ for each group.

a

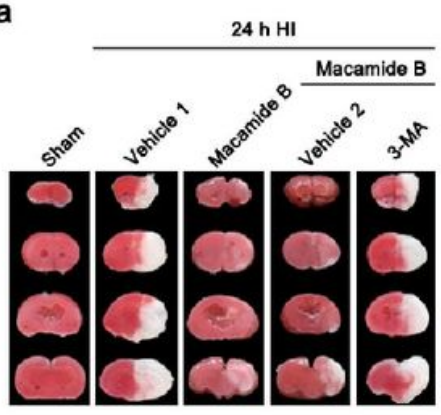

b

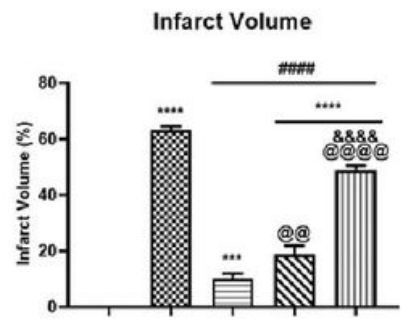

ㅁ Sham

as Hitvehicle 1

C. Hithacamide 8 .

G. HI+Macamide B+3-MA

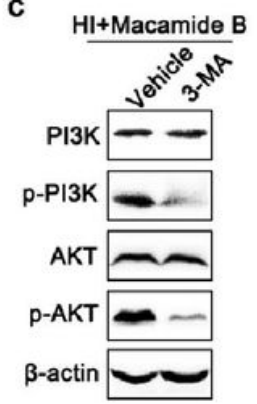

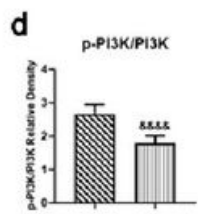

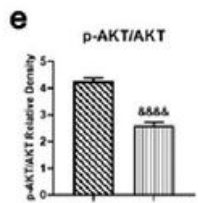

a. HitMacamide B*vehicle a H1+Macamide B+3-MA

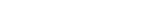

(1) WI+Macamide B+Vehich
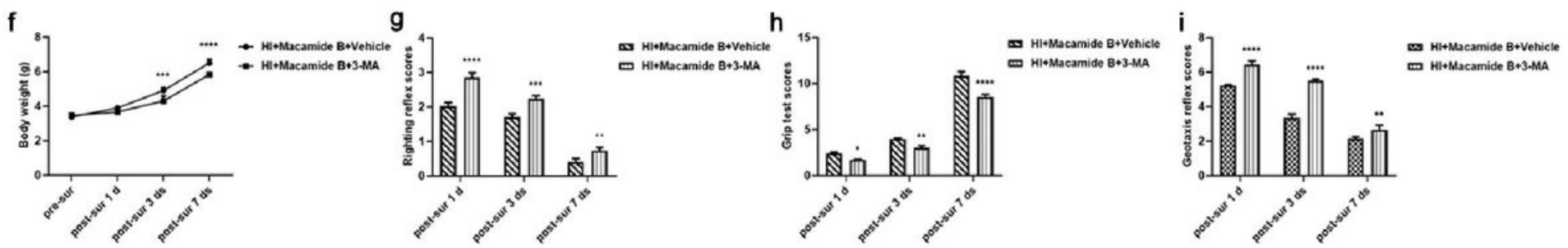


\section{Figure 9}

The effect of 3-MA on PI3K-AKT signaling pathway and HIBD neonatal mice pretreated with macamide $\mathrm{B}$. a Representative pictures of coronal brain sections stained with TTC $24 \mathrm{~h}$ after HIBD. b Quantitative analysis results of cerebral infarct volume in pups. Vehicle 1 is the vehicle of macamide $B$, vehicle 2 is the vehicle of 3-MA. Compared with the vehicle 2 group, the cerebral infarct area of the pups treated with 3MA increased significantly. $\mathrm{n}=6$ for each group. $\mathrm{c}$ Representative western blots and quantification data of $\mathrm{d} \mathrm{p}$-PI3K and e p-Akt protein in the ipsilateral cerebral hemisphere were detected $24 \mathrm{~h}$ after HIBD. Macamide B pretreatment significantly inhibited the expression of p-PI3K and p-Akt proteins in the HI brain. $\mathrm{n}=6$ for each group. On postoperative days 1, 3, and 7, 3-MA reversed macamide B-pretreated HIBD pups' increased $f$ body weights and exacerbated neurobehavioral damage in the $g$ righting reflex, $h$ negative geotaxis, and i grip tests compared to the macamide $B+$ vehicle 2 groups. ${ }^{\star \star \star \star} p<0.0001$ vs. sham group; $* \star \star p<<0.01$ vs. sham group; \#\#\#\#p<0.0001 vs. vehicle1 group; @@p< 0.01 vs. macamide $B$ group; @@@@ 0.0001 vs. macamide B group; \&\&\&\&p<0.0001 vs. macamide B + vehicle 2 group.
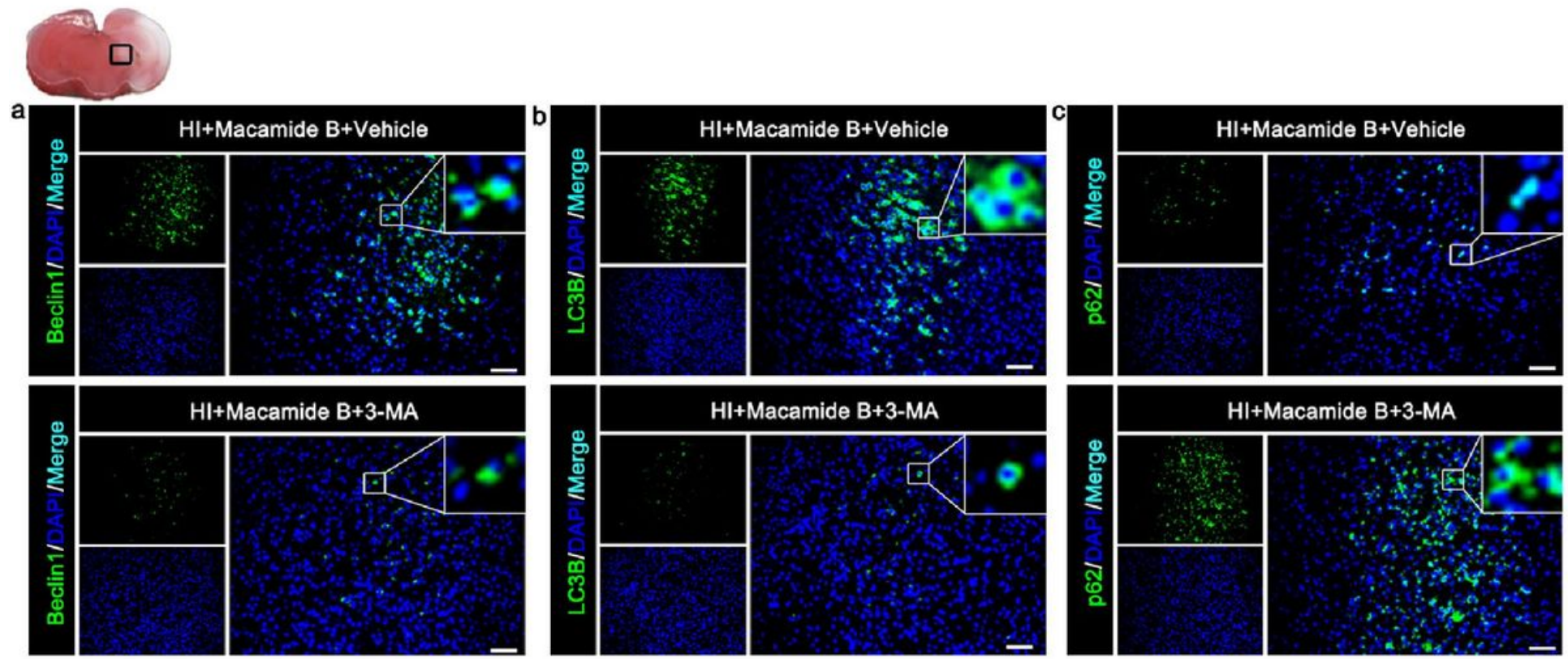

\section{Figure 10}

Representative micrographs of a Beclin 1 (green fluorescence), b LC3B (green fluorescence), and c p62 (green fluorescence) positive cells in the ipsilateral cortex of HIBD neonatal mice showed the effect of 3MA intervention on autophagy in HIBD neonatal mice. Blue fluorescence represented DAPI, and merge was the combination of the target cell and the corresponding nucleus. The vehicle is the vehicle of 3-MA. Compared with macamide B + vehicle control group, the number of a Beclin1 and $b$ LC3B positive cells in the 3-MA intervention group increased significantly, while the number of $c$ p62 positive cells decreased significantly. The brain slice in the upper left corner shows the position of immunofluorescence staining (small black box). $\mathrm{n}=8$ for each group. Scale bar $=100 \mu \mathrm{m}$ 
a

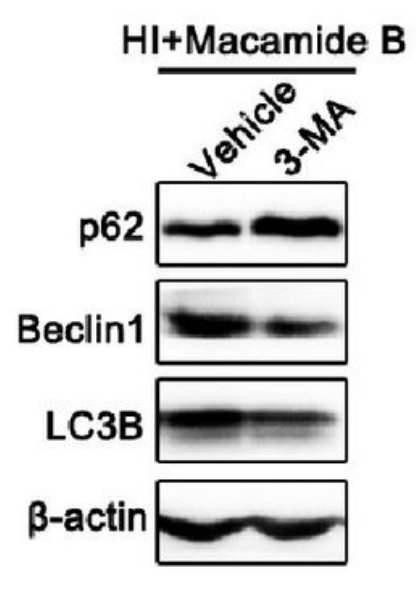

c

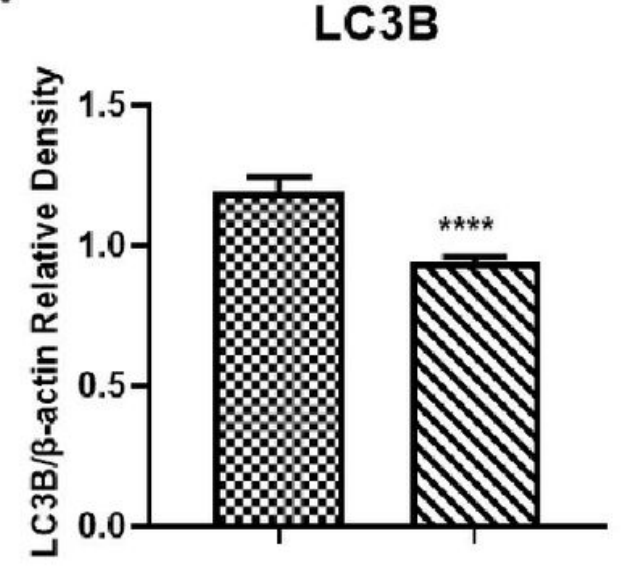

b

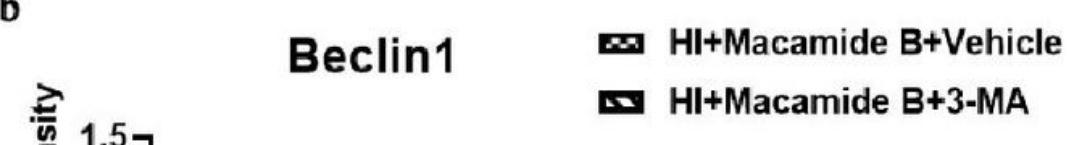

\section{Figure 11}

a Representative western blots and quantification data of b Beclin1, c LC3B, and d p62 protein in the ipsilateral cerebral hemisphere were detected $24 \mathrm{~h}$ after HIBD. It shows the effect of 3-MA intervention on autophagy-related proteins in HIBD neonatal mice pretreated with macamide $B$. Vehicle is the vehicle of 3MA. Compared with the macamide B + vehicle control group, the Beclin1 and LC3B protein expression levels in the 3-MA intervention group decreased significantly, and the p62 protein expression level increased significantly. ${ }^{* \star *} \mathrm{p}<0.0001$ vs. sham group; ${ }^{* \star *} \mathrm{p}<0.0001$ vs. sham group. $\mathrm{n}=6$ for each group. 

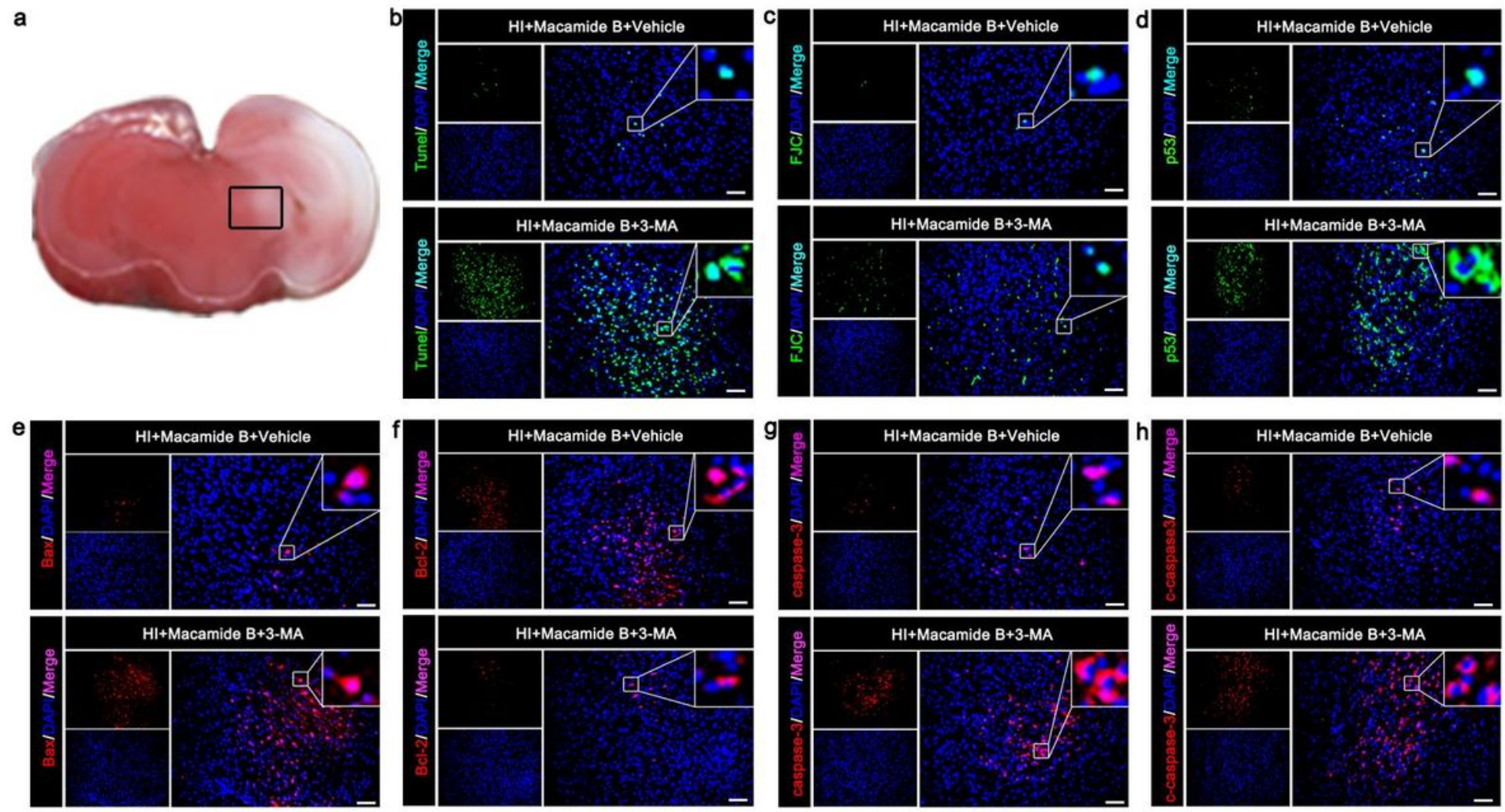

Figure 12

Representative micrographs of b TUNEL-positive cells (green), c FJC-positive cells (green), and d p53 (green), e Bax (red), f Bcl-2 (red), g caspase-3 (red), and h cleaved caspase-3 (red) positive cells in the ipsilateral cortex of HIBD newborn mice showed the effect of 3-MA intervention on apoptosis in HIBD newborn mice. Blue fluorescence represented DAPI, and merge was the combination of the target cell and the corresponding nucleus. The vehicle is the vehicle of 3-MA. Compared with the macamide $\mathrm{B}+$ vehicle group, the number of $\mathrm{p} 53$, Bax, caspase-3, and cleaved caspase-3 positive cells in the 3-MA intervention group increased significantly, while the number of Bcl-2 positive cells decreased significantly. a The brain slice shows the position of immunofluorescence staining (small black box). $\mathrm{n}=8$ for each group. Scale bar $=100 \mu \mathrm{m}$. 


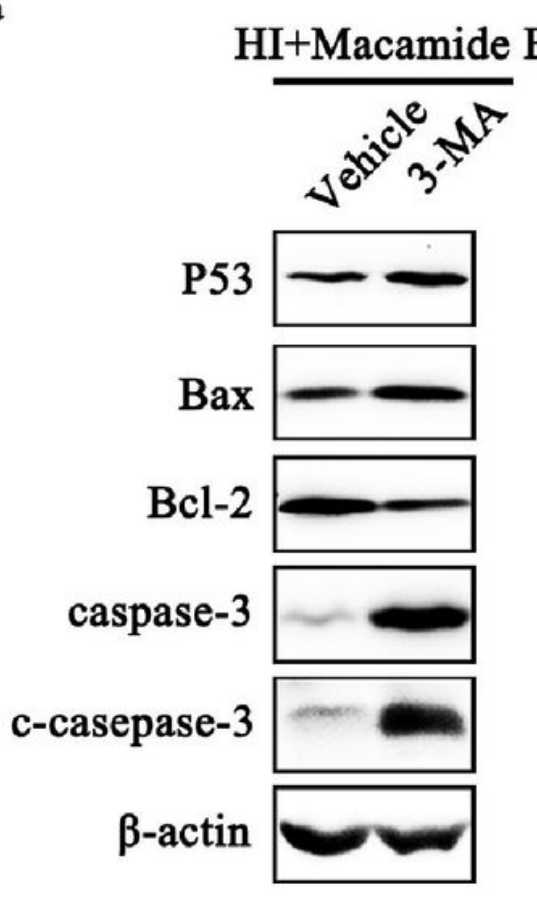

b

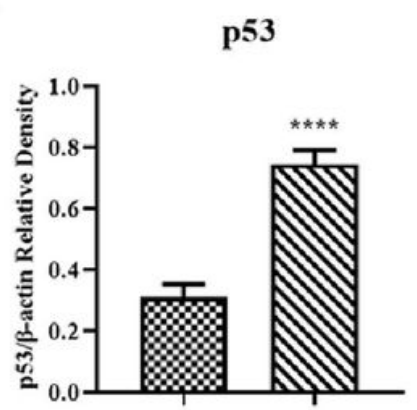

d

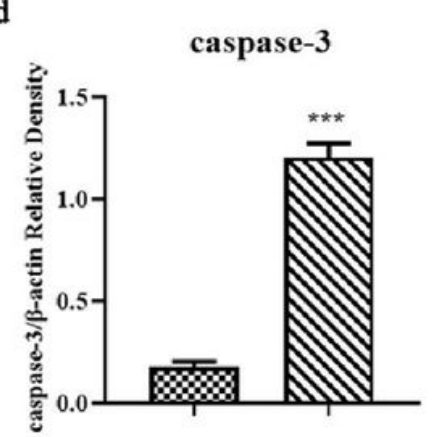

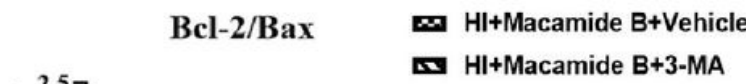

\section{Figure 13}

a Representative western blots and quantification data of b p53, c Bcl-2 / d Bax, e caspase-3, and f cleaved caspase- 3 in the ipsilateral cerebral hemisphere were detected $24 \mathrm{~h}$ after HIBD. Compared with the macamide $B+$ vehicle control group, the $\mathrm{p} 53$, caspase -3 , and cleaved caspase- 3 protein expression levels in the 3-MA intervention group were significantly increased, and the Bcl-2 / Bax ratio was significantly decreased. ${ }^{\star \star \star \star} p<0.0001$ vs. sham group; ${ }^{\star \star \star} p<0.0001$ vs. sham group. $n=6$ for each group. 


\section{Macamide B \\ a. \\ HMP}

.

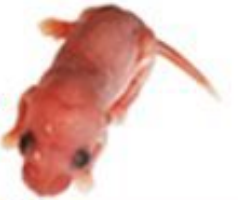

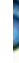
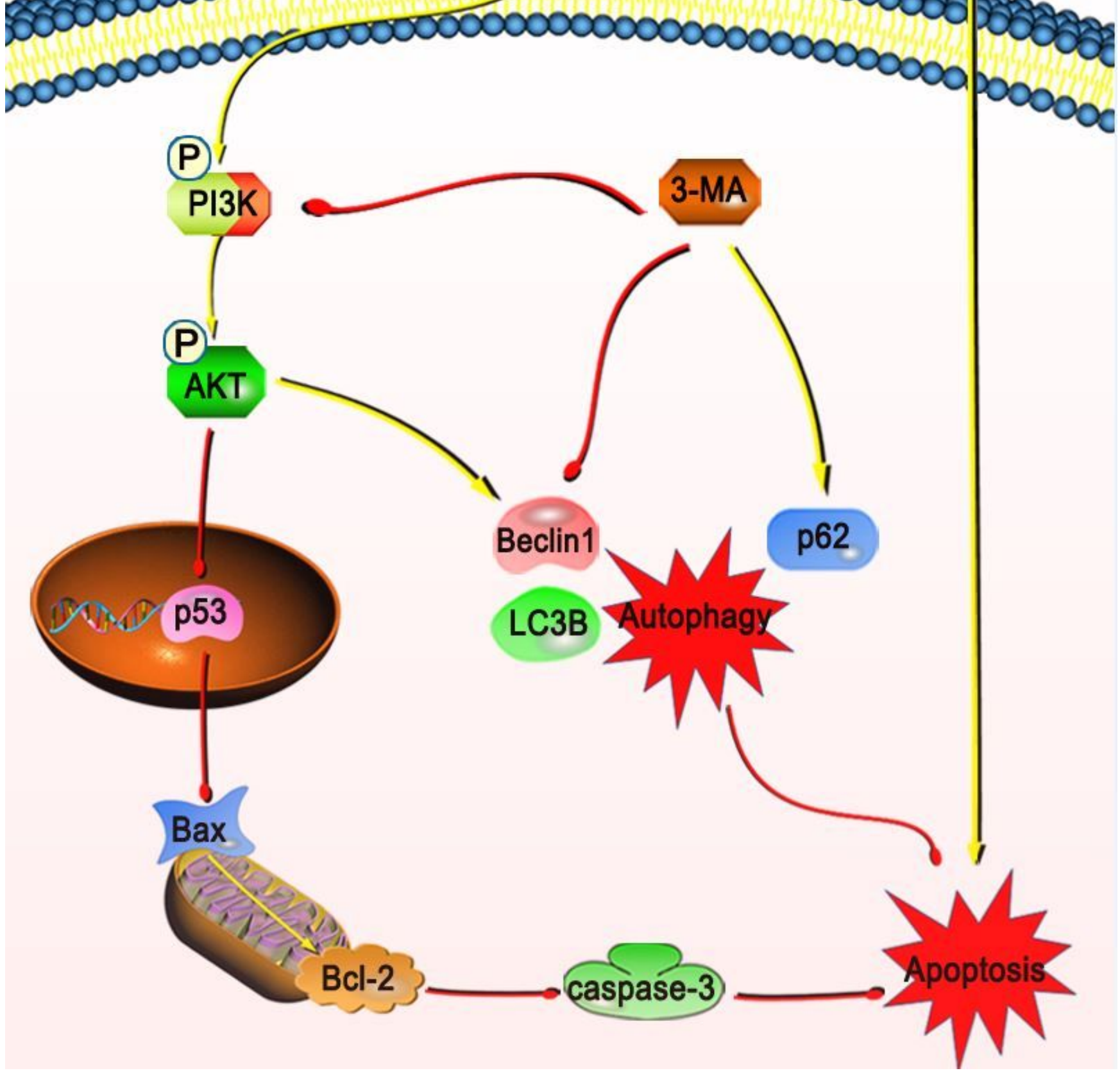

Figure 14

The neuroprotective mechanism of macamide B pretreatment on HIBD newborn mice. 